\title{
Differential V2-directed Antibody Responses in Non- human Primates Infected with SHIVs or Immunized with Diverse HIV Vaccines
}

\section{Susan Zolla-Pazner ( $\sim$ Susan.Zolla-Pazner@mssm.edu )}

Icahn School of Medicine at Mount Sinai https://orcid.org/0000-0002-0750-2666

\section{Svenja Weiss}

Icahn School of Medicine at Mount Sinai https://orcid.org/0000-0003-4136-0566

Vincenza Itri

Icahn School of Medicine at Mount Sinai

\section{Ruimin Pan}

New York University School of Medicine

\section{Xunqing Jiang}

New York University School of Medicine

Christina Luo

New York University School of Medicine

Lynn Morris

National Institute for Communicable Diseases https://orcid.org/0000-0003-3961-7828

Delphine Malherbe

Oregon Health \& Science University https://orcid.org/0000-0001-6416-6227

Philip Barnette

Oregon Health \& Science University

Jeff Alexander

PaxVax Corporation https://orcid.org/0000-0001-7066-3780

\section{Xiang-Peng Kong}

New York University Langone Medical Center

Nancy Haigwood

Oregon Health \& Science University

Ann Hessell

Oregon Health \& Science University

\section{Ralf Duerr}

New York University https://orcid.org/0000-0001-9569-9237

\section{Article}


Keywords: V2 Ab response, SHIV infection, vaccine regimen

Posted Date: October 5th, 2021

DOI: https://doi.org/10.21203/rs.3.rs-829888/v1

License: (c) (1) This work is licensed under a Creative Commons Attribution 4.0 International License. Read Full License 


\section{Abstract}

V2p and V2i antibodies (Abs) that are specific for epitopes in the V1V2 region of the HIV gp120 envelope (Env) do not effectively neutralize HIV but mediate Fc-dependent anti-viral activities that have been correlated with protection from, or control of HIV, SIV and SHIV infection. Here, we describe a novel molecular toolbox that allows the discrimination of antigenically and functionally distinct polyclonal V2 $\mathrm{Ab}$ responses. We identified different patterns of $\mathrm{V} 2 \mathrm{Ab}$ induction by SHIV infection and three separate vaccine regimens that will aid in fine tuning an optimized immunization protocol for inducing $V 2 p$ and V2i Abs. We observed no, or weak and sporadic V2p and V2i Abs in non-vaccinated Tier 1 and Tier 2 SHIV-infected NHPs, but in contrast, strong V2p and/or V2i Ab responses after immunization with a V2targeting vaccine protocol using a prime/boost regimen with gp120 DNA and a V1V2-scaffold protein. The V2-targeting vaccine protocol is superior to both natural infection and to immunization with whole Env constructs for inducing functional V2p- and V2i-specific responses. Strikingly, levels of V2-directed Abs correlated inversely with Abs specific for gp120 and peptides of V3 and C5. These data demonstrate that a V1V2-targeting vaccine have advantages over the imprecise targeting of SIV/SHIV infections and of whole Env-based immunization regimens for inducing a more focused functional V2p- and V2i-specific Ab response.

\section{Introduction}

RV144 is the only Phase $2 b / 3$ clinical vaccine trial to date to demonstrate modest but significant efficacy in preventing HIV infection ${ }^{1}$. Subsequent studies of specimens from RV144 volunteers indicated that the only primary, independent correlate of reduced risk (CoR) was a robust level of non-neutralizing Abs binding to a fusion protein containing the first and second variable regions (V1V2) of gp120, a domain in the envelope (Env) glycoprotein of HIV-1 2,3,4. Later experiments demonstrated a significant inverse CoR with binding to $\mathrm{V} 2$ peptides ${ }^{5}$. These studies generated the hypothesis that Abs directed against V1V2 contributed to the reduced incidence in HIV infections in vaccinees $1,2,3,4,6$.

The findings from RV144 continue to be debated $7,8,9$, especially following the failure of the recent HVTN 702 vaccine trial in South Africa to demonstrate efficacy ${ }^{10}$, although the HVTN 702 immunization regimen differed in several aspects from RV144 ${ }^{11}$. Nonetheless, the RV144 CoR with V1V2 Abs has been buttressed by similar conclusions emanating from several studies of immunized NHPs in which protection, control and/or delayed infection with SIV or SHIV were correlated with strong Ab responses to the V1V2 domain of gp120 (reviewed in ${ }^{8}$ ). These findings have given rise to the hypothesis that efficient induction of V2-specific Abs would aid in preventing HIV infection.

Three V1V2 domains form the apex of the Env trimer, which upon Env binding to CD4, undergoes extreme conformational changes allowing access to the coreceptor binding site ${ }^{12}$; this is a requisite process for the initiation of infection. The structurally stabilized V1V2 domain forms a five-stranded $\beta$-barrel ${ }^{13}, 14$, in the prefusion trimer, however, its C-strand (V2C) exists in both a-helical and $\beta$-strand configurations 
depending on the V1V2 sequence, its molecular environment, and structural constraints $13,14,15,16,17,18$, 19,20 .

Many human anti-V1V2 monoclonal Abs (mAbs) have been isolated and the epitopes recognized by these mAbs have been classified into four families: V2i, V2p, V2q and V2qt ${ }^{8,21} 15,22$. V2i-specific mAbs, including mAbs $830 \mathrm{~A}$ and 2158 , recognize $\mathrm{V} 2$ when its $\mathrm{V} 2 \mathrm{C}$ region is in a $\beta$-strand configuration; the epitope region of these $\mathrm{V} 2 \mathrm{i} \mathrm{mAbs}$ is discontinuous, highly conformational, and overlaps the $\mathrm{a} 4 \beta \mathrm{B}$ integrinbinding motif ${ }^{13,21}$. V2p mAbs, including mAbs $\mathrm{CH} 58$ and CAP228-16H, were isolated from a recipient of the RV144 vaccine regimen and an infected individual, respectively, and target the V2C strand region as an a-helix and extended coil ${ }^{16,19,23}$. The epitope region recognized by V2q mAbs, such as mAbs PG9 and PG16, includes two N-linked glycans, e.g., N156 and N160, and consists of the V2C in its $\beta$ strand configuration $18,24,25,26,27$, while the epitope region recognized by V2qt mAbs, such as PGT145 and PGDM1400, is located at the axial center of the Env trimer ${ }^{28,29}$.

V2q and V2qt mAbs display broad and potent virus neutralizing activities $29,30,31,32,33$ as well as other Ab effector functions (reviewed in ${ }^{7}$ ). As yet, no vaccine has induced broad and potent V2q or V2qt Abs in humans or any animal models. While V2p and V2i mAbs are poor neutralizers, they are effective at mediating various Fc-dependent anti-viral activities $20,34,35,36,37$. An ever-increasing number of studies indicates that Abs with anti-viral functions mediated by the Fc-fragment of Abs are effective against various viral infections, including HIV, SIV, SHIV, influenza, SARS-CoV-2 and herpes 38, 39, 40, 41, 42, $43,44,45,46,47,48,49,50,51,52$.

Several vaccine regimens have been shown to induce Abs reactive with V2 peptides or V1V2-scaffold protein in rabbits, non-human primates (NHPs), ${ }^{22,53,54}$ and humans ${ }^{2,3,4}$. However, little is known about the fine specificities of the vaccine-induced polyclonal V2 Ab responses, the relative levels of V2p vs. V2 $i$ Abs in serum, or how robust V2-specific Ab responses can best be induced. Furthermore, no study has directly investigated V1V2 responses in SHIV-infected vs. vaccinated NHPs. Therefore, we undertook studies to elucidate the fine specificity and function of V2p and V2i Abs in SHIV-infected and vaccinated NHPs with the goal of providing valuable insights for the design of vaccines for both prevention of infection and therapeutic control of viral replication.

Here, we systematically probed the nature of the V1V2 Ab response of 20 SHIV-infected and 25 immunized rhesus macaques using one of three different vaccine regimens. We used a newly developed panel of antigens (Ags) that presents V2 or V1V2 in various molecular settings in which V2C assumes ahelical and/or $\beta$-stranded configurations $13,15,16,17,18,55$ that react with V2p and/or V2i mAbs, respectively. The results show that in NHPs infected for 3-4 months with Tier 1 or Tier 2 SHIVs, essentially no V2p or V2i Abs were induced. Immunizations with Env/Gag DNA or a recombinant adenovirus vector along with gp120 or gp140 induced V2p Abs. In contrast, animals receiving Env DNA and V1V2-scaffold protein immunogens mounted targeted functional $A b$ responses of both the V2 $p$ and V2 $i$ types, 
underscoring the influence of vaccine composition for developing strength, specificity and functionality of $\mathrm{V} 2 \mathrm{Ab}$ responses.

\section{Results}

\section{V2-specific mAbs distinguish between HIV-1 Env peptides, proteins and V1V2-scaffold proteins. To} assess their antigenic nature, three gp120 proteins, five cyclic V2 (cV2) peptides, three linear V2 peptides ${ }^{56}$ and eleven V1V2-fusion proteins were tested for reactivity with five V2p-, four V2i-, one V2q-and one V2qt-specific mAbs. Anti-V3 mAb 447-52D and linear V3 and C5 peptides were used as controls. The sequences of V2p epitopes of several of the reagents used in this study are shown in Supplementary Table S1. The results, shown in Figure 1a, from multiplex bead Ab binding assays using these reagents, are represented as area-under-the-curve (AUC) values that were derived from titration curves (Supplementary Figure S1). The data indicate that V2p and V2i mAbs display extensive cross-reactivity and different patterns of binding reactivity: V2p mAbs recognize cV2 peptides from many clades, but are non-reactive with three of four V1V2-1FD6 molecules. For example, strong cross-reactivity is seen in the data in Figure 1a by mAb V2p CAP228-16H with reagents from three clade $C$ strains (ZM53, 1086, 97ZA012), two clade A strains (MG505 and 92RW020), two clade AE strains (92TH023 and A244) and one clade B strain (CaseA2) although, as shown in Supplementary Table S1, strains C/1086 and A/MG505 differ in sequence by $32 \%$ ( 6 of 19 positions in V2) and yet have similar and strong reactivity with CAP228-16H. These data support crystallographic data indicating that V2p mAbs recognize the V2C strand in its alpha-helical configuration ${ }^{19,20}$. Two of these V2p mAbs (mAbs CH58 and CH59) were derived from individuals immunized with the RV144 vaccine regimen consisting of immunogens from clades $B$ and $A E{ }^{19}$, while the other three V2p mAbs (CAP228-19F, -3D.1, and -16H) were derived from a clade $\mathrm{C}$-infected individual ${ }^{20}$. Notably, these five $\mathrm{mAbs}$ are cross-reactive with antigens derived from clades $A, C$ and $A E$, but not with clade $B$.

In contrast, V2i mAbs, all of which were derived from clade B-infected individuals $57,58,59,60$, react with V1V2-1FD6 Ags, but bind poorly, if at all, with cV2 peptides. They are highly cross-clade reactive with Ags from clades $A, B, C$, and $A E$. This broad cross-reactivity is consistent with their recognition of the conserved $\beta$-barrel conformation of the V1V2 domain $13,15,21$. Statistical analyses comparing binding of $V 2 p$ and V2i mAbs to cV2 peptides and V1V2-1FD6 proteins reflect these differential activities. Binding of V2p mAbs to cV2 peptides was significantly stronger than binding to V1V2-1FD6 $(p<0.0001)$, while binding of V2i mAbs was significantly stronger to V1V2-1FD6 than binding to cV2 peptides $(p=0.0006)$ (Figure 1b).

Interesting exceptions were noted with particular antigen/mAb pairs: there was no significant difference in binding of either V2p or V2i mAbs to clade AE-based cV2 peptides or V1V2-1FD6 proteins suggesting that the $\mathrm{V} 2 \mathrm{C}$ region of strain $\mathrm{AE} / 92 \mathrm{TH} 023$ transitions between an a-helix and a $\beta$-strand (Figure $\mathbf{1 b}$ and Figure $\mathbf{2 b}$ ). In contrast, the $\mathrm{V} 2 \mathrm{C}$ region exists solely as an a-helix/random coil in $\mathrm{cV} 2_{\mathrm{C} / 1086}$ and $\mathrm{cV} 2_{\mathrm{A} / \mathrm{MG} 505}$ (Figure 2b). Another exception is V2p mAb CAP228-16 H which can react with three V1V2- 
1FD6 scaffold proteins from clade $\mathrm{C}^{16}$ indicating that $\mathrm{V} 2 \mathrm{C}$ regions in some clade $\mathrm{C}$ strains can form ahelices and/or $\beta$-strands.

Identification of V1V2 antigens for defining the reactivities of V2p and V2i Abs. To examine the relative presence of a-helix/random coil and/or $\beta$-strand configurations in V1V2-or V2-peptides in solution, CD spectra were generated and fitted using the CD-FIT program

(https://www.ruppweb.org/Xray/comp/cdfit.htm) (Figure 2a and 2b). Analyses of various cV2s from clades $A, B, C$, and AE identified $81-100 \%$ of the secondary structures as a-helix/random coils, and $0-19 \%$ as $\beta$-strands. In contrast, when fused with an $\mathrm{N}$-terminal Ig leader sequence and $\mathrm{C}$-terminal avi- and histags (V1V2-tags), the a-helix/random coil and $\beta$-strand proportions of V1V2 or V2 Ags studied ranged from $54-71 \%$ and $29-46 \%$, respectively. These findings are consistent with those of Rao et al. and i et al., where additional $\mathrm{cV} 2$ peptides exhibited a propensity to form random coil/alpha-helices ${ }^{16,61}$.

Given the preferential specificity of V2p mAbs for cV2 peptides and of V2i mAbs for V1V2-1FD6 scaffold proteins, reactivity with $\mathrm{cV} 2$ peptides was used as a hallmark for the presence of polyclonal V2p Abs in plasma from infected and immunized animals, while V1V2-1FD6 molecules were used as a marker to identify V2i plasma Abs. Gp120, V1V2-tags and V1V2-gp70 reagents reacted with both V2p and V2i mAbs (Figure 1a) and therefore were not used to identify V2p and V2i Abs. This paradigm is represented diagrammatically in Figure $2 \mathrm{c}$ showing the alternative a-helix and $\beta$-strand configurations of the V2C region, the mAbs that preferentially bind to each, and the Ags that exist in either or both configurations.

\section{Patterns of plasma Ab binding to V2 and other Env epitopes differ between infected and immunized} rhesus macaques. We performed experiments to determine the relative levels of these Abs induced by infection of NHPs with either clade C Tier 1 SHIV $_{1157 \text { ipEL-p }}$ or clade C Tier 2 SHIV $_{1157-\text { ipd3N4 }}$ and by vaccination of NHPs with one of three distinct immunization regimens. All immunizations consisted of co-immunization or prime/boost strategies in M. mulatta with the same adjuvant (Adjuplex) as shown in Table l: the "DNA + gp120" group is comprised of animals in which DNA encoding for gp $160_{\text {AE/92TH023 }}$

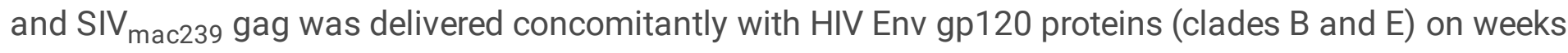
0, 4, 12 and 20 62; the "SAd7 + gp140" group received simian adenovirus 7 (SAd7) carrying genes for HIV clade $C$ Env gp $150_{\mathrm{C} / 1086}$ on weeks 0 and 4, and gp $140_{\mathrm{C} / 1086}$ and GBV-C E2 proteins on weeks 4 and 16 53; and the "DNA + V1V2-scaffold" group was immunized with HIV clade C Env gp120 ${ }_{\text {C/ZM53 }}$ DNA at

weeks 0,8 , and 20 along with three V1V2-scaffold recombinant proteins, $\mathrm{V} 1 \mathrm{~V} 2_{\mathrm{C} / \mathrm{ZM} 53^{-}} \mathrm{F} \mathrm{KK}, \mathrm{V} 1 \mathrm{~V} 2_{\mathrm{C} / \mathrm{ZM} 109^{-}}$ TTB and $\mathrm{V} 1 \mathrm{~V} 2_{\mathrm{AE} / \mathrm{A} 244}-2 \mathrm{~J} 9 \mathrm{C} 22,62,63$.

To identify the patterns of $\mathrm{V} 2 \mathrm{Ab}$ specificities induced by infection, specimens were obtained at necropsy from the animals infected with Tier 1 SHIV $_{1157 \text { ipEL-p }}$ and Tier 2 SHIV $_{1157-\text { ipd3N4 }}$ at weeks 18 and 11 post last challenge, respectively. For immunized animals, blood was drawn two weeks after the last immunization. Plasma specimens were initially screened for binding to the Ag panel described in Figure 1a using a plasma dilution of 1:200 (Figure 3 and Supplementary Figure S2). These data are 
complemented by titration of plasma using dilutions of 1:100-1:150,000 for SHIV-infected animals (Supplementary Figure S3a-b) and for “DNA + gp120" immunized NHPs (Supplementary Figure S3c).

Plasma from the SHIV-infected animals and animals in all the immunized groups reacted with gp120 glycoproteins from clades $A, B$, and C; they also reacted strongly with $\mathrm{V}_{\text {consc }}$ even in the animals that had not been exposed to clade $C$ immunogens, i.e., the "DNA + gp120" group. Antibodies specific for C5, which are among the highest titered Abs in infected humans 5,64 , were elicited in infected NHPs. Notably, among the immunized NHPs, only those in the "DNA + gp120" group mounted a C5 Ab response (Figure 3 and Supplementary Figure S3c). These data suggest that C5 Abs are poorly induced by the

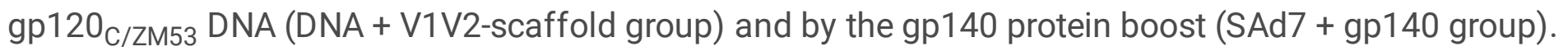
Given the conservation of $\mathrm{C} 5$, it is probable that if these animals, immunized with a clade $\mathrm{C}$ gp140, could have mounted a $\mathrm{C} 5 \mathrm{Ab}$ response, they would have reacted with the $\mathrm{C} 5_{\mathrm{consC}}$ peptide. Further examination of the data indicates that strong responders had high levels against all Ags tested, and conversely, for weak responders (Supplementary Figure S2).

There is a remarkable paucity of V1V2-specific Ab responses in the Tier 2- and Tier 1-SHIV-infected animals 11 and 18 weeks after the last challenge dose, respectively (Figure 3); this stands in contrast to the uniform Ab responses to gp120 from clades A, B and C and to a V3 peptide heterologous to the infecting strains. These data suggest that if there was a substantial $A b$ response to $V 2$ epitopes in the infected NHPs, it would have been detected given the similarities at key residues between the V2p regions found in the two clade $C$ V2 peptides studied here and the similar region in the SHIV strains used to infect the NHPs (Supplementary Table S1). In addition, it is unlikely that mutations that might have occurred in the $\mathrm{V} 2$ region after 18 weeks of SHIV infection would account for a loss or gain of cross-reactive Abs.

All immunized animals displayed Abs to Ags carrying V2 or V1V2. The most extensive V1V2 responses were obtained in the "DNA + V1V2-scaffold" immunized group which displayed Abs reactive with all 19 of the V2 and V1V2 Ags tested. The next most V2-responsive group was the "SAd7 + gp140" group (reactive with 12/19 V2 and V1V2 Ags), followed by the "DNA + gp120" group (reactive with 11/19 Ags).

Interestingly, V2p Ab responses in all three immunized NHP groups were highly reactive with the linear peptides spanning V2(AA166-185) AE/A244 $_{\text {and V2(AA172-191) }}$ AE/A244 which corresponds to the region covering the V2C-strand that includes the residues at positions 169 and 181 that were the key signatures of escape from vaccine efficacy in the RV144 clinical trial. The V2p Abs were cross-reactive with peptides from heterologous strains and clades. For example, plasma from the "SAd7 + gp140" group which received HIV immunogens from clade $\mathrm{C}$ strain 1086 reacted with V2 peptides from the homologous and heterologous strains $\mathrm{C} / 1086$ and $\mathrm{C} / \mathrm{ZM} 109$, and with $\mathrm{V} 2$ peptides from the heterologous clade $\mathrm{AE}$ strain 92TH023 (Figure 3). Though only a single V2 peptide from clades A and B were tested, there was poor or no reactivity with these peptides in either infected or immunized animals, including those in the "DNA + gp120" group which received the gp120 ${ }_{\mathrm{B} / \mathrm{MN}}$ protein. In contrast to the reactivity with V2C-strand peptides, there was little or no Ab reactivity in the plasma of infected or immunized NHPs with a peptide that

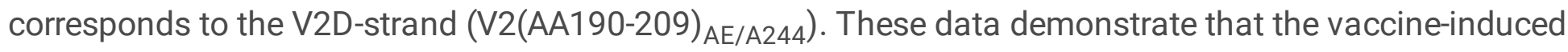


Abs from all three vaccine regimens tested focused the $\mathrm{Ab}$ response on the $\mathrm{V} 2 \mathrm{C}$ strand in its ahelix/random coiled configuration.

As noted, the primary variable in RV144 associated with the reduced risk of infection was Abs reactive with V1V2-gp70 ${ }_{\mathrm{B} / \mathrm{CaseA} 2}$ 2, 3, 4 , a reagent recognized by both V2i mAbs and V2p mAbs (Figure 1a). A V2ispecific $\mathrm{mAb}$ was also associated with the partial control of SHIV $_{\text {Bal }}$ infection ${ }^{65}$. Therefore, it is noteworthy that among the five groups of animals studied, only the "DNA + V1V2-scaffold" group mounted a $\mathrm{V} 2 \mathrm{i}$ Ab response as shown by the reactivity of the plasma from animals in this group with V1V2-1FD6 constructs.

V1V2-scaffold-induced V2p and V2i-specific antibodies mediate Fc-dependent functions. To dissect the functions of vaccine-induced Abs that are specific for V2p and/or V2i, two assays were used: (a) binding of the $\mathrm{C} 1 \mathrm{q}$ component of complement to the $\mathrm{Fc}$ fragment of Abs bound to $\mathrm{cV} 2$ peptides $(\mathrm{cV} 2 \mathrm{C} / 1086$ and $\mathrm{CV} 2_{\mathrm{AE} / 92 \mathrm{TH} 023}$ ) or to V1V2-1FD6 scaffold proteins (V1V2 ${ }_{\mathrm{C} / \mathrm{ZM} 109^{-1 F D 6} \text { and V1V2 }}$ B/YU2 ${ }^{-1 F D 6}$ ) which denotes the initiation of complement activation, and (b) ADCP initiated by beads coated with the same Ags. Both of these functions are associated with non-neutralizing control of HIV and SIV infection ${ }^{66,67}$. Results from these assays are represented as AUC values (Figure 4) derived from titration curves (Supplementary Figure S4).

C1q binding by V2p and V2i Abs. Despite the fact that V2p-specific binding Abs were detected in the plasma of all three groups of immunized animals (Figure 3 and $4 a$ ), V2p Abs recognizing $c V 2$ peptides bound C1q poorly, if at all (Figure $\mathbf{4 b}$ and Supplementary Figure S4a). These data indicate either that the V2p Abs are of an isotype that does not activate complement or, more likely, that binding to peptides does not permit the aggregation of Abs needed to initiate the complement cascade.

In contrast, when C1q binding to Abs was measured using V1V2-1FD6 Ags, the animals receiving the "DNA + V1V2-scaffold" regimen were the only immunized animals with V2i-specific Abs and the only animals able to bind C1q. The C1q binding was observed with V1V2-1FD6 Ags derived from the homologous C/ZM109 immunogen used in the "DNA + V1V2-scaffold" group, and from a heterologous clade, B/YU2 (Figure 4b and Supplementary Figure S4a).

Plasma of both Tier 1 and Tier 2-infected animals had strong responses to gp120 but low levels of V2p Abs and no detectable V2i Abs (Figure 3). C1q binding by gp120-specific Abs from Tier 2-infected animals was low but detectable whereas no plasma V2p or V2i Abs from this group were able to bind to C1q. Plasma from Tier 1-infected animals showed only weak binding of C1q to V2p Abs, but no C1q binding to either gp120 or V2i specific Abs. (Supplementary Figure S4a). In summary, of the Abs tested in the plasma of the two groups of SHIV-infected NHPs and the three groups of immunized animals, primarily those receiving the "DNA + V1V2-scaffold" vaccine regimen responded with V2 Abs able to activate the complement cascade. 
ADCP mediated by V2 Abs. As previously reported, all three HIV-1 Env vaccine regimens were able to induce Abs with Fc-dependent effector functions including ADCC against $\mathrm{SHIV}_{\mathrm{SF} 162 \mathrm{p3}}{ }^{22,62,63}$, ADCP

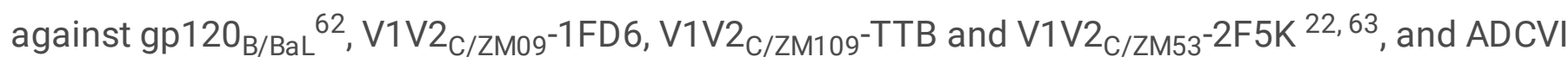
against SHIV-1157ipEL-p ${ }^{53}$.

Detection of ADCP activity was observed in the plasma of animals immunized with the "DNA + V1V2scaffold" regimen and was seen with beads coated with V1V2-1FD6 proteins but not with beads coated with cV2 peptides (Figure $4 c$ and Supplemental Figure S4b). Neither infected NHPs nor animals vaccinated with the other two immunization regimens displayed ADCP activity. Thus, only the V2targeting immunization regimen induced $A b s$ that could mediate both $\mathrm{C} 1 \mathrm{q}$ binding and ADCP activities since only immunization with the V2-targeting regimen (DNA + V1V2-scaffolds) induced V2i Abs, only this group of immunized animals displayed ADCP activity, and the ADCP activity correlated strongly with the presence of V2i Abs (Figure 4 and see below).

\section{Full parameter analysis reveals Ab signatures that are distinct between NHPs infected with SHIVs or immunized with different regimens. The compiled data from all $45 \mathrm{NHP}$ plasma samples, generated by} the multiplex $\mathrm{Ab}$ binding, $\mathrm{C} 1 \mathrm{q}$ and $\mathrm{ADCP}$ assays are summarized in the cluster heatmap shown in Figure 5. The data for each animal are shown in a separate row. The data from Ab binding, C1q binding, and ADCP assays using different Ags are depicted in each column.

Hierarchical clustering of the animals reveals that the NHPs in the three immunization groups (pink) cluster separately from the infected animals (blue, see dendrogram on left of heatmap) and that there is an intermixing of Tier 1 and Tier 2 SHIV-infected animals as well as an intermixing of the animals receiving the "DNA + gp120" and "SAd7 + gp140" vaccine regimens. The animals in the "DNA + V1V2scaffold" group are clearly distinct (Figure $5 \mathbf{a}$ and $\mathbf{b}$ ). Additional principal component analyses show the separate clustering of each of the five groups of animals, with the "DNA + V1V2-scaffold" group being the most unique (Figure 5c, Supplementary Figure S5).

Cluster analysis of the binding data revealed by the dendrogram at the top left of Figure $5 \mathbf{a}$ shows four families of Ags composed of (i) gp120, V3 and C5; (ii) primarily V2 peptides; (iii) primarily V1V2-1FD6; and (iv) primarily V1V2-gp70. This representation of the binding data indicates that (a) the "DNA + V1V2scaffold" group shows decreased binding to gp120, V3 and C5 (family i), (b) the immunized groups but not the infected groups have mounted a strong response to V2p Ags (family ii), (c) only the "DNA + V1V2scaffold" group makes Abs that recognize the V2i Ags (family iii), and (d) all groups make Abs that recognize Ags that co-exist in the V2p and V2i configurations, although this reactivity is weakest in infected animals (family iv).

\section{V1V2 immune profiles cluster separately from immunodominant responses to gp120, C5 and V3}

epitopes. To examine the interrelationships between Ab binding and biological activity, multi-parameter linear correlation analyses were performed (Figure 6, Supplementary Figure S6). Three clusters were identified showing strong positive correlations (outlined with boxes in yellow = cluster 1 , red $=$ cluster 2 , 
and orange $=$ cluster 3 , Figure 6 ), and an additional two clusters show negative correlations (outlined by boxes in black = cluster 4 and blue = cluster 5 in Figure 6). Cluster 1 shows strong positive correlations between binding of Abs to gp120 glycoproteins and the consensus C V3 peptide. Cluster 2 shows strong positive correlations between Abs binding to V1V2-gp70 and V2 peptides, i.e., Ags that bind primarily to V2p Abs. Cluster 3 shows strong positive correlations primarily between Abs binding to V1V2-1FD6 (which bind V2i Abs) and Abs that mediate ADCP and complement activation. This correlation analysis supports the data shown above (Figures 4 and $5 a$ ) indicating that the only group of animals whose plasma Abs displayed C1q binding and ADCP was the "DNA + V1V2-scaffold" group in which the vaccine response resulted in both $\mathrm{V} 2 p$ and $\mathrm{V} 2 \mathrm{i}$ Abs.

Clusters of negative correlations were also identified: cluster 4 shows strong negative correlations between Abs that display V1V2-1FD6-binding, C1q-binding and ADCP activity vs. Abs that bind to gp120 and V3. Cluster 5 shows negative correlations of anti-C5 Abs vs. Abs that mediate C1q, ADCP and that bind to V1V2 Ags. This correlation analysis supports the data shown in Figures $\mathbf{4}$ and $\mathbf{5 a}$ in which, again, it was the "DNA + V1V2-scaffold" group which showed the strongest V1V2 responses, C1q binding and $A D C P$, but the poorest Ab levels to gp120, V3 and C5. -

A correlation network analysis of data from the multiplex Ab bead binding assay (Figure 7) highlights the anti-V2 Ab responses which are strongly positively correlated with each other but are negatively correlated with Ab responses to gp120, V3 and C5.

\section{Discussion}

Identifying virus epitopes that induce protective Abs is the key to designing effective vaccines. The rapid identification of the receptor binding domain of the SARS-CoV-2 Spike protein and the production of highly effective vaccines based on multiple platforms in 2020 has been remarkable. In contrast, the HIV field has labored for over 40 years to accomplish a similar feat and remains confronted with the hurdles imposed by a trimeric envelope protein on which the sites of vulnerability are occluded by glycans, along with extensive mutational variation and extreme conformational flexibility. This presents a conundrum in terms of which epitope(s) should be and can be targeted to elicit a protective immune response to HIV.

Several studies in the literature suggest that the V1V2 domain of Env contains epitopes worth targeting with a vaccine. These include quaternary epitopes targeted by such Abs as PG9 and PGT145 (reviewed in ${ }^{68}$ ), as well as epitopes that are not dependent on the trimeric structure of Env, such as those targeted by V2i mAbs such as 2158 and 697, and by V2p mAbs such as CH58 and CAP228-19F 15, 19, 20,69. Data support the hypothesis that Abs directed at the V1V2 domain of gp120 contributed to a reduced risk of HIV infection in humans $2,3,4,5,70$, and similarly have been implicated in the reduction and control of infection of NHPs with SIV $69,71,72,73,74,75,76,77$. Additional studies in macaques infected with various strains of SHIV also appear to support this hypothesis but are not definitive ${ }^{53,78,8}$ ). Using these studies as a foundation, and samples from vaccinated NHPs and non-vaccinated SHIV-infected NHPs, we developed a molecular toolkit of curated antigens representing multiple epitopes within V1V2, gp120 
proteins from various clades, and V3 and C5 peptides to characterize and parse immune responses in NHPs. In particular, we sought to identify Ab responses induced by three different immunization regimens and by infection with Tier 1 and Tier 2 SHIVs in order to determine the extent to which these responses targeted the V1V2 domain, and to compare the patterns of V1V2 Abs for quantity, function and fine specificity.

While broadly neutralizing V2q and V2qt Abs appear in $<2 \%$ of HIV infected individuals ${ }^{79}$, they have yet to be induced by any vaccines tested in humans or animals. In contrast, V2p and V2i Abs are present in the serum of $30-84 \%$ of infected individuals $80,81,82,83$ and have been induced with vaccines in humans ${ }^{1}$, 84,85 . As we have shown here and previously ${ }^{22}$, Abs of these specificities can be induced with vaccines in NHPs, and they are cross-clade reactive, and exhibit anti-viral activities, although, at best, are poorly neutralizing. The current study describes methods for measuring the presence and levels of Abs specific for V2p and V2i epitopes in plasma, for assessing the anti-viral activities of these two types of Abs. Here, we have also evaluated an effective approach that selectively induces V2p and V2i Abs.

Heretofore, reagents have not been available to easily distinguish between the various types of V2 Abs in plasma. For this, we developed a panel of Ags bearing the V2 sequences for the V1V2 domains from several clades and strains and characterized them in terms of their ability to cross-react with 11 mAbs that target the V2p, V2i, V2q and V2qt epitopes (Figure 1a and Supplementary Table SI). The reactivity of these Ags in a multiplex $A b$ binding assay indicated that $\mathrm{V} 2 p$ mAbs bound to V2 peptides which present the V2C-strand as an a-helix/coil, ${ }^{15}, 23,86$ while V2i mAbs bind preferentially to V1V2-1FD6 Ags in which V2C is most frequently present as a $\beta$-strand as part of the V1V2 $\beta$-barrel ${ }^{13,14}$. As we show, there are exceptions: reagents based on clade AE present an unusual pattern: the V1V2-1FD6 scaffold protein and the $\mathrm{V} 2$ peptides derived from clade $\mathrm{AE}$ can assume both the a-helix/coil and $\beta$-strand conformations as shown by their ability to be bound by both V2p and V2i mAbs (Figure 1b). This may relate to the unusual ability of the clade $\mathrm{AE} \mathrm{gp} 120_{\mathrm{A} 244}$ to induce in humans particularly strong V2-specific responses directed to both $\mathrm{V} 2 \mathrm{p}$ and $\mathrm{V} 2 \mathrm{i}$ 2,87, and the ability of the "DNA + V1V2-scaffold" group of immunized NHPs (which were boosted with three V1V2-scaffolds, including V1V2 ${ }_{\mathrm{AE} / \mathrm{A} 244}-2 \mathrm{J9C}$ ) to induce both V2p and V2i Abs (Figure 3).

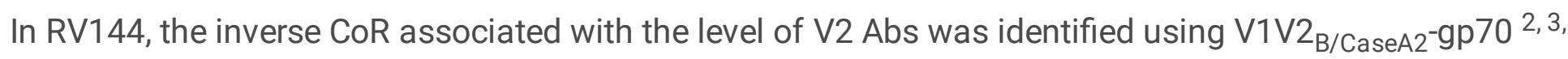
${ }^{4}$. Since V1V2-gp70 reagents react with both V2p and V2i Abs (Figure 1a), the primary RV144 CoR with

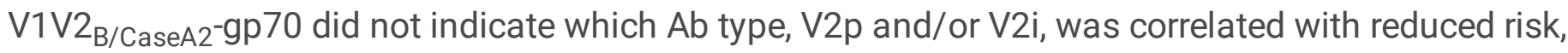
although the subsequently identified CoR with V2 peptides supports the hypothesis that V2p Abs were involved ${ }^{5}$. Since passive immunization of NHPs with V2i mAb 830A followed by challenge with SHIV $\mathrm{BaL}_{\mathrm{L}}$ led to reduced virus levels in plasma and peripheral blood mononuclear cells and decreased viral DNA in lymphoid tissues, there is evidence that V2i Abs can contribute to control of infection ${ }^{65}$. Additionally, though most studies of V2 correlates of protection in NHPs against SIV and SHIV did not test for V2i Abs, Abs specific for $\mathrm{V} 2$ peptides were correlated with protection and/or control (reviewed in ${ }^{8}$ ). Two additional active immunization studies support a protective role for V2 Abs: higher V1V2-specific binding antibody 
titers correlated with viral control in the SAd7 + gp140 vaccine group ${ }^{53}$, and in a challenge study of the nine animals in the "DNA + V1V2" group, four of nine NHPs $(p=0.0497)$ immunized with a V2-focusing regimen showed an absence of plasma vRNA, cell-associated VDNA in PBMCs, and lymphoid-associated virus over a nine-week period following the final challenge dose with the heterologous Tier $1 \mathrm{SHIV}_{\mathrm{BaL} . \mathrm{P} 4}$ ${ }^{62}$. In the latter study, the predominant V2 Ab response was directed at V2 peptides, with little or no response to V1V2-1FD6, demonstrating a predominant effect of V2p Abs. However, the V2 responses did not differ in the tight controllers compared with the non-controllers, leaving open the question of the role of the V2p-directed Abs using this regimen.

The Luminex data presented above show that all three immunization regimens examined here induced V2p Abs but only the "DNA + V1V2-scaffold" immunization regimen gave robust Ab responses to both V2p and V2i Ags (Figure 3). Importantly, all three immunization regimens induced cross-clade reactive Ab responses, with reactivity to Ags carrying V1V2 and V2 segments from clades $A, B, C$ and AE. The broad cross-reactive binding of the vaccine-elicited V2 Abs underscores shared antigenic features ${ }^{88}$ and the common structure that previous studies have shown the V1V2 domain to assume ${ }^{13,14}$. Notably, boosting with the V1V2-scaffold proteins elicited only weak responses to gp120, V3 and C5 (Figures 3 and 5). This is an indication that the use of the V1V2-scaffold proteins focused the immune response on the V2 region, which was the intent of the design of this immunization regimen $22,37,55$.

It is noteworthy that, with the sole exception of Abs to $\mathrm{C} 5$, immunization by the three vaccine regimens induced responses that were equal to or stronger than those induced by infection (Figure 3 and 5). This indicates that vaccines can induce immune responses that are qualitatively and quantitatively different compared to infection, and this is especially true for V2 Ab responses. Since infection does not lead to subsequent protection, as shown by the ability of HIV to superinfect already infected individuals (reviewed in ${ }^{89}$ ), effective HIV vaccines will have to "do better than Nature". The Ab responses elicited by HIV vaccines will need to preferentially target epitopes that elicit functional, cross-reactive, and durable $A b$ responses. The fact that native Env trimers presented on virions in the course of infection are not able to induce these features suggests that epitope-targeting immunogens, such as the ones tested in the "DNA + V1V2-scaffold" group, may be particularly useful constructs.

In the data presented, only the V2-directed immunization regimen induced Abs with the Fc-mediated effector functions of ADCP and complement activation. Assessing the combined datasets of $A b$ binding and functionality, an inverse immune pattern was observed for $\mathrm{V} 2$ responses compared to those for gp120, V3, and C5 (Figures 5, 6 and 7). These data suggest that Abs to immunodominant epitopes including V 3 and $\mathrm{C} 5$ that are induced by whole Env vaccines may divert immune responses from less immunogenic epitopes such as those responsible for the induction of functional and potentially protective $A b$ responses, including those specific for $V 2 p$ and $V 2 i$.

We and others have shown that both V2i and V2p mAbs can mediate ADCP and ADCC 20, 22, 37, 90, and that both $A D C P$ and ADCC have been correlated with reduced risk of HIV and SIV and SHIV infection in 
NHPs ${ }^{8}$. Indeed, both ADCC and ADCP were analyzed in the monkeys receiving the "DNA + V1V2-scaffold" vaccine and the responses were robust and long-lasting 22,63 .

Taken together, the findings of this study suggest that the patterns of induced V1V2-specific Abs differ significantly between SHIV infection and immunization in NHPs, that immunization can induce more potent binding responses to this region of Env than infection, and that effective induction of functional V2p and V2i Abs benefits from targeting the V1V2 domain with V1V2-scaffold proteins. Moreover, since V1V2 Abs display a variety of Fc-mediated anti-viral functions that correlate with protection from HIV, SIV and SHIV, data showing that V2p and V2i Abs can easily be induced is of particular significance. Given that these data indicate that the levels of V1V2-specific Abs can be enhanced by epitope-targeting vaccines, they constitute an important consideration in terms of moving the field toward the development of an efficacious HIV vaccine. Finally, the type of comprehensive analysis of Ab fine specificity targeting Env epitopes will be important for discriminating between immunization protocols and perhaps for establishing go/no-go criteria for clinical trials.

\section{Methods}

Multiplex bead Ab binding assay. Antigens included recombinant gp $120_{\mathrm{C} / \mathrm{ZM} 53}$, gp $120_{\mathrm{B} / \mathrm{YU} 2}$ and gp120 ${ }_{\mathrm{A} / \mathrm{MG} 505}$ from Immune Tech (New York, NY). To ensure sufficient coupling of peptides to the beads, all peptides used bore an N-terminal 6x Lys-Gly (KG)-linker. Peptides purchased from GenScript (Piscataway, NJ) included: clade $C$ consensus V3 linear peptide (NNTRKSIRIGPGQTFYATGDIIG), cyclic V2 ${ }_{\text {AE/92TH023 }}$ peptide (CSFNMTTELRDKKQKVHALFYKLDIVPIEDNTSSSEYRLINC), cV2 $\mathrm{C/ZM109}$ peptide (CSFNITTDVKDRKQKVNATFYDLDIVPLSSSDNSSNSSLYRLISC), $\mathrm{cV} 2_{\mathrm{C} / 1086}$ peptide (CSFKATTELKDKKHKVHALFYKLDVVPLNGSSSSGEYRLINC), cV2 $2_{\mathrm{B} / Y{ }_{2}}$ peptide (CSFNITTSI RDKVQKEYALFYNLDVVPIDNASYRLISC), cV2 A/MG505 $_{5}$ peptide (CSFNMTTELRDKKQKVYSLFYR

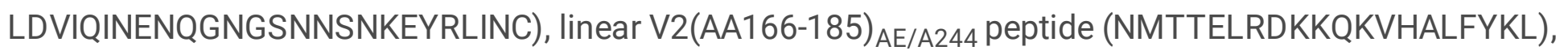

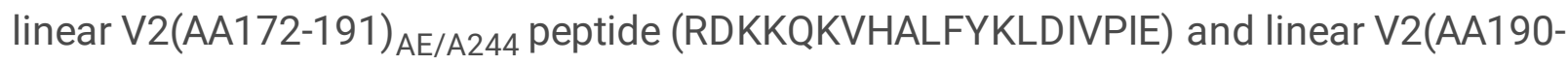
209) AE/A244 $_{\text {peptide (IEDNNDNSKYRLINCNTSVI). C5 }}$ /ZM109 linear peptide (VEIKPLGIAPTEAKRRVVQREKR) was purchased from BioPeptide (San Diego, CA). V1V2-scaffold proteins bearing V1V2 domain inserts from a variety of HIV-1 strains and clades included V1V2-1FD6 24, 55, V1V2tags and V1V2-gp70 reagents provided by B. Haynes and J. Peacock (Duke University) and V1V2 ${ }_{B / C a s e A 2^{-}}$ gp70 provided by A. Pinter (Rutgers University).

Antigens were covalently coupled to magnetic beads using a two-step carbodiimide reaction with the xMAP Antibody Coupling (AbC) Kit according to manufacturers' instructions (Luminex, Austin, TX).

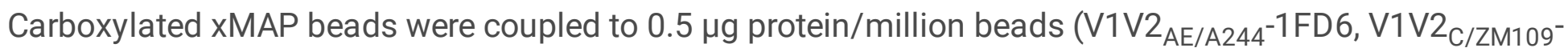
$1 \mathrm{FD} 6$ and $\mathrm{V} 1 \mathrm{~V} 2_{\mathrm{B} / \mathrm{YU} 2^{-1}} \mathrm{FD} 6$ ) or $1 \mu \mathrm{g}$ protein/million beads (all peptides and $\mathrm{V} 1 \mathrm{~V} 2_{\mathrm{A} / \mathrm{MG} 505^{-1 F D 6}}$ ) or $4 \mu \mathrm{g}$ protein/million beads (all gp120s, all V1V2-tags and -gp70, and BSA). The coupled beads were counted, diluted to a concentration of 500,000 beads $/ \mathrm{ml}$ and stored at $4^{\circ} \mathrm{C}$ for up to 1 month prior to use. The 
concentrations used on the beads were established on the basis of the reactivity with mAbs which were tested at concentrations ranging from $5-10 \times 10^{-6} \mu \mathrm{g} / \mathrm{ml}$. Beads coupled to BSA served as negative controls. A cocktail of mAbs composed of multiple V2i (697, 830A, 1393A), V2p (CH58), V3 (3869) and C5 $(670,1331 \mathrm{~A}) \mathrm{mAbs}{ }^{19}, 57,59,60,91,92$ was used as a positive control. The conditions of the assay were established so that the readings used were from the mid-point (most sensitive part) of the sigmoidal titration curve, rather than using saturating conditions. This allowed inter-experimental standardization.

Each Ag was bound to a bead region with a different fluorescent profile. PE fluorescence was measured using a Luminex FlexMAP3D device with XPONENT 4.2 software. NHP plasma were first screened for reactivity at a 1:200 dilution, and for titrations, dilutions ranged from 1:100-150,000. Samples were tested in duplicates and results shown as mean fluorescence intensity (MFI).

Titration curves and scatter plots were generated in GraphPad Prism 7.03. AUC values were based on titration curves and divided by a denominator giving values between 0 and 15 .

Circular dichroism measurements. Circular dichroism (CD) spectra were recorded on a Jasco J-1500 spectrometer equipped with a temperature controller using $1 \mathrm{~mm}$ length cells and a scan speed of 4 $\mathrm{nm} / \mathrm{min}$. The spectra were averaged over eight scans with the background subtracted according to the analogous experimental conditions. The peptides and proteins were prepared at 12.5-50 $\mu \mathrm{M}$ in 0.1x PBS $(\mathrm{pH} 7.4)$ and measured at $25^{\circ} \mathrm{C}$. Helical propensity titrations were conducted using $12.5-50 \mu \mathrm{M}$ peptide in $50 \%$ v/v 2,2,2-trifluoroethanol (TFE) (Sigma-Aldrich, St. Louis, MO). CD data were fitted using the CD-FIT program (https://www.ruppweb.org/Xray/comp/cdfit.htm) and pie charts were generated based on the percentage of secondary structure (a-helix, random coil, or $\beta$-sheet) calculated by CD-FIT software.

Rhesus macaque immunizations and challenges. Experimental groups of adult rhesus macaques were infected with either a Tier 1 or Tier 2 SHIV, or immunized with one of three immunization regimens. The infected groups each consisted of ten animals that received four weekly intrarectal challenges with 5,000 $\mathrm{TCID}_{50}$ of Tier 2 clade C SHIV ${ }_{\text {ipd3N4 }}{ }^{93}$ or three weekly intrarectal challenges with 8,000 TCID 50 of Tier 1 clade C SHIV 1157ipEL-p $^{53}$. Final blood draws occurred at 11 and 18 weeks after the last challenge dose, respectively. Twenty-five animals were immunized using three different vaccine regimens (Table I). One group of nine animals was co-immunized with env-gag DNA (36 $\mu \mathrm{g}$ of gp160 ${ }_{92 \text { TH028_F8 }}$ and V1R SIV gag OPT) and $100 \mu \mathrm{g}$ each of gp120 proteins from clade B MN and CRF01_AE A244 delivered intramuscularly (i.m.) (“DNA + gp120" group). Four immunizations were delivered at weeks 0, 4, 12 and $20{ }^{62}$. The second immunized group consisting of ten animals received a prime of the Simian Adenovirus 7 vectors (SAd7) carrying $g p 150_{C / 1086}$ and $g a g_{S / V m a c 239}$ transgenes $\left(1 \times 10^{11}\right.$ virus particles, i.m. and $0.5 \times 10^{11}$ virus particles, intranasally) at weeks 0 and 4 and a protein boost with soluble trimeric gp $140_{\mathrm{C} / 1086}$ and GBV-C E2 protein (25 $\mu \mathrm{g}$ each) at weeks 4 and 16, i.m. ${ }^{53}$. The third group of six animals was co-immunized with a total of $36 \mu \mathrm{g} \mathrm{gp} 120_{\mathrm{C} / \mathrm{ZM} 53}$ DNA intradermally and three V1V2-scaffolds including V1V2 ${ }_{\mathrm{C} / \mathrm{ZM} 53^{-}}$

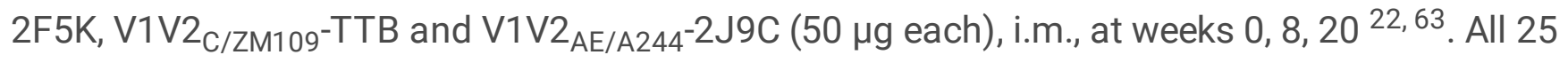


immunized animals received their protein vaccines together with Adjuplex. Data shown for all three immunization groups are for blood specimens drawn two weeks after the last boost.

C1q binding ELISA to measure complement activation. C1q binding to V2- and gp120-specific Abs was measured by ELISA as described previously ${ }^{56}$. Briefly, Immulon 4HBX 96-well plates (Thermo Scientific, Waltham, MA) were coated with $2 \mu \mathrm{g} / \mathrm{ml}$ of the following Ags: $\mathrm{cV} 2_{\mathrm{C} / 1086}, \mathrm{cV} 2_{\mathrm{AE} / 92 \mathrm{TH} 023}, \mathrm{~V} 1 \mathrm{~V} 2_{\mathrm{C} / \mathrm{ZM} 109^{-}}$ $1 F D 6, \mathrm{~V} 1 \mathrm{~V} 2_{\mathrm{B} / \mathrm{Yu} 2}-1 \mathrm{FD} 6, \mathrm{gp} 120_{\mathrm{C} / \mathrm{ZM} 109}$ or gp120 $\mathrm{B}_{\mathrm{VU} 2}$. Plates were incubated at $4^{\circ} \mathrm{C}$ overnight, washed with $0.05 \%$ bovine serum albumin (BSA)/PBS, and blocked in PBS/7.5\% BSA for $1.5 \mathrm{hr}$ at room temperature. Plasma samples were heat inactivated at $56^{\circ} \mathrm{C}$ for $30 \mathrm{~min}$, titrated at 2-fold dilutions ranging from $50 \mathrm{x}$ to 400x and added to the plate. After $1.5 \mathrm{hr}$ incubation at room temperature, plates were washed and incubated with $10 \mu \mathrm{g} / \mathrm{ml}$ of $\mathrm{C} 1 \mathrm{q}$ (Sigma-Aldrich, St. Louis, MO) for $1.5 \mathrm{~h}$ at room temperature with shaking at $500 \mathrm{rpm}$. The C1q was diluted in PBS/ 0.5\% BSA/0.05\% Tween 20. After washing, bound Abs were detected with $10 \mu \mathrm{g} / \mathrm{ml}$ mouse monoclonal IgG1 anti-human C1q-HRP (Santa Cruz, Dallas, TX) diluted in PBS/0.5\% BSA/0.05\% Tween 20 and incubated for $1 \mathrm{hr}$ at room temperature with shaking at $500 \mathrm{rpm}$. After washing, plates were developed with 3,3',5,5'-tetramethylbenzidine (TMB) substrate (Thermo Scientific, Waltham, MA) followed by $1 \mathrm{~N}$ hydrochloric acid and read at $450 \mathrm{~nm}$ on a BioTek PowerWave HT plate reader. Assays were standardized with positive controls (mAb CAP228-16H for V2p Ags, mAb 830A for V2i, and gp120 Ags), and a negative control anti-anthrax PA-specific mAb 3685 was included with each assay. Background values from pre-bleed specimens were subtracted from the values obtained with specimens drawn two weeks after the last immunization or 18/11 weeks after infection, and the area under the curve (AUC) was determined.

ADCP. Antibody-dependent cellular phagocytosis (ADCP) was performed by measuring the uptake of HIV Env Ag-coated microspheres by the monocytic THP-1 cell line (ATCC), as described previously ${ }^{63}$. Briefly, 5 $\mu \mathrm{g}$ of $\mathrm{Ag}\left(\mathrm{cV} 2_{\mathrm{C} / 1086}, \mathrm{CV} 2_{\mathrm{AE} / 92 \mathrm{TH} 023}, \mathrm{~V} 1 \mathrm{~V} 2_{\mathrm{C} / \mathrm{ZM} 109}-1 \mathrm{FD} 6\right.$ or V1V2 $\left.{ }_{\mathrm{B} / \mathrm{YU} 2}-1 \mathrm{FD} 6\right)$ was biotinylated using EZ-Link Sulfo-NHS-LC-biotin (Thermo Scientific, Waltham, MA) and then conjugated to $1 \mu \mathrm{m}$ fluorescent neutravidin beads (Thermo Scientific, Waltham, MA) according to the manufacturer's instructions. Conjugated beads were washed and resuspended in PBS/0.1\% BSA. A total of $1.8 \times 10^{6}$ beads was aliquoted per well in round bottom 96-well plates. Plasma was titrated using five-fold dilutions and added to the beads, followed by incubation for $2 \mathrm{hr}$ at $37^{\circ} \mathrm{C}$ and a washing step. A total of $2.5 \times 10^{4} \mathrm{THP}-1 \mathrm{cells}$ was added to each well and incubated overnight at $37^{\circ} \mathrm{C}$. Phagocytosis was measured by flow cytometry using a BD LSR II Fortessa equipped with an HTS plate reader and analyzed by FCS Express 7 Flow Research Edition (De Novo Software; Pasadena, CA). The gating strategy is supplied in Supplementary Fig S7. ADCP scores were calculated by multiplying the percentage of bead-positive cells by mean fluorescent intensity (MFI) and dividing by a denominator giving values between 0 and 10 . AUC values were calculated from the titration curves as described for $\mathrm{C} 1 \mathrm{q}$ binding.

Software scripts and visualization. Normalized heatmaps were generated using the complexheatmap and tidyverse packages in program R x64 version 4.0.2 and RStudio version 1.3.959 ${ }^{94,95}$. Normalizations were done for each assay type/Ag combination (parameter). Principal component analyses were done 
with factoextra and tidyverse packages using prcomp function based on singular value decomposition in $\mathrm{R}$ and RStudio.

For generating the correlogram, the corrplot and RColorBrewer packages in R and RStudio using hierarchical clustering (hclust) were used. Dendrograms were calculated using the dendPlot function and hclust method, or as implemented in the complexheatmap packages in R. Annotations were done using thecomplexheatmap package.

The correlation network diagram was generated in undirected mode in R and RStudio using ggraph, igraph and tidyverse packages, with clustering based on the graphopt layout. Edges are weighted according to $r$ values. Edges are only shown if $p<0.05$, and nodes without edges were removed. Nodes are sized according to the $r$ values of the connecting edges.

Statistical analyses. D'Agostino and Pearson normality tests were performed with the software GraphPad Prism 7.03 (La Jolla, CA). The statistical comparison of groups was done using the two-tailed Wilcoxon matched pairs signed rank test (mAb analyses). Multiparameter pairwise correlation analyses were done using Pearson's correlation as data were in a linear range, following bivariate distribution. Correlation coefficients $r$ and $p$ values were calculated in GraphPad Prism and a $p$ value $<0.05$ was considered significant. Multiplicity adjustments for $p$ values were performed with the Benjamini-Hochberg method in $\mathrm{R}$ and $\mathrm{R}$ Studio using the data.table and tidyverse packages ${ }^{94,95}$.

\section{Declarations}

\section{ETHICS STATEMENT}

All animals were housed and all animal experiments were conducted at Oregon National Primate Research Center. All procedures were performed according to rules and protocols approved by the Institutional Animal Care and Use Committee (IACUC) at Oregon Health \& Science University. At the end of the study, animals were not euthanized, but instead all animals were released from assignment and returned to the colony at Oregon National Primate Research Center.

\section{DATA AVAILABILITY}

All relevant data are available from the corresponding author upon reasonable request.

\section{ACKNOWLEDGEMENTS}

This work was supported in part with funds from the National Institute of Allergy and Infectious Diseases, P01 Al100151 (PIs: X-P Kong and S. Zolla-Pazner), R01 Al145655 (PI: X-P Kong), R01 Al112546 (PI: M. K. Gorny), P51 OD011092 (N. Haigwood), P01 Al078064 (N. Haigwood), U42 OD010426 (N. Haigwood), R44 Al091546 (J. Alexander), R01 Al122953-05 (PI: R. Duerr) and R01 Al104387 (L. Morris), as well as the South African Medical Research Council (L. Morris) and the Department of Medicine, Icahn School of Medicine at Mount Sinai (S.Zolla-Pazner). 


\section{AUTHOR CONTRIBUTIONS}

S.W., J.A., N.L.H., A.J.H., M.K.G. and S.Z-P. conceived and designed experiments. S.W., V.I., R.P., D.C.M., P.B. performed the experiments. X.J. and C.L. produced protein immunogens. L.M. provided the mAbs CAP228-19F, -3D.1 and -16H. R.P. performed the circular dichroism experiments. S.W., R.P., X-P K., A.J.H., R.D. and S.Z-P. analyzed and interpreted data. S.W. and S.Z-P. wrote the manuscript; and all authors read, edited, and approved the manuscript.

\section{COMPETING INTERESTS}

The authors declare no competing interests.

\section{References}

1. Rerks-Ngarm, S. et al. Vaccination with ALVAC and AIDSVAX to prevent HIV-1 infection in Thailand. N Engl J Med 361, 2209-2220 (2009).

2. Haynes, B.F. et al. Immune-correlates analysis of an HIV-1 vaccine efficacy trial. N Engl J Med $366,1275-1286$ (2012).

3. Zolla-Pazner, S. et al. Vaccine-induced IgG antibodies to V1V2 regions of multiple HIV-1 subtypes correlate with decreased risk of HIV-1 infection. PLoS One 9, e87572 (2014).

4. Zolla-Pazner, S. et al. Analysis of V2 antibody responses induced in vaccinees in the ALVAC/AIDSVAX HIV-1 vaccine efficacy trial. PLoS One 8, e53629 (2013).

5. Gottardo, R. et al. Plasma IgG to linear epitopes in the V2 and V3 regions of HIV-1 gp120 correlate with a reduced risk of infection in the RV144 vaccine efficacy trial. PLoS One 8, e75665 (2013).

6. Zolla-Pazner, S. et al. Vaccine-induced human antibodies specific for the third variable region of HIV-1 gp120 impose immune pressure on infecting viruses. EBioMedicine 1, 37-45 (2014).

7. Duerr, R. \& Gorny, M.K. V2-specific antibodies in HIV-1 vaccine research and natural infection: controllers or surrogate markers. Vaccines (Basel) 7, 82 (2019).

8. Zolla-Pazner, S., Alvarez, R., Kong, X.P. \& Weiss, S. Vaccine-induced V1V2-specific antibodies control and or protect against infection with HIV, SIV and SHIV. Curr Opin HIV Aids 14, 309-317 (2019).

9. Desrosiers, R.C. Protection against HIV acquisition in the RV144 trial. J Viro/ 91, e00905-00917 (2017).

10. Gray, G.E. et al. Vaccine efficacy of ALVAC-HIV and bivalent subtype C gp120-MF59 in adults. N Engl J Med 384, 1089-1100 (2021). 
11. Zolla-Pazner, S., Michael, N.L. \& Kim, J.H. A tale of four studies: HIV vaccine immunogenicity and efficacy in clinical trials. Lancet HIV 8, e449-e452 (2021).

12. Wang, H. et al. Cryo-EM structure of a CD4-bound open HIV-1 envelope trimer reveals structural rearrangements of the gp120 V1V2 loop. Proc Natl Acad Sci U S A 113, e7151-e7158 (2016).

13. Pan, R., Gorny, M.K., Zolla-Pazner, S. \& Kong, X.P. The V1V2 region of HIV-1 gp120 forms a fivestranded beta barrel. J Viro/ 89, 8003-8010 (2015).

14. Gorman, J. et al. Structures of HIV-1 Env V1V2 with broadly neutralizing antibodies reveal commonalities that enable vaccine design. Nat Struct Mol Biol 23, 81-90 (2016).

15. Spurrier, B., Sampson, J., Gorny, M.K., Zolla-Pazner, S. \& Kong, X.P. Functional implications of the binding mode of a human conformation-dependent V2 monoclonal antibody against HIV. J Virol 88, 4100-4112 (2014).

16. Wibmer, C.K. et al. Common helical V1V2 conformations of HIV-1 Envelope expose the alpha4beta7 binding site on intact virions. Nat Commun 9, 4489 (2018).

17. Munro, J.B. et al. Conformational dynamics of single HIV-1 envelope trimers on the surface of native virions. Science 346, 759-763 (2014).

18. McLellan, J.S. et al. Structure of HIV-1 gp120 V1/V2 domain with broadly neutralizing antibody PG9. Nature 480, 336-343 (2011).

19. Liao, H.X. et al. Vaccine induction of antibodies against a structurally heterogeneous site of immune pressure within HIV-1 envelope protein variable regions 1 and 2. Immunity 38, 176-186 (2013).

20. van Eeden, C. et al. V2-directed vaccine-like antibodies from HIV-1 infection identify an additional K169-binding light chain motif with broad ADCC activity. Cell Rep 25, 3123-3135 (2018).

21. Mayr, L.M., Cohen, S., Spurrier, B., Kong, X.P. \& Zolla-Pazner, S. Epitope mapping of conformational V2-specific anti-HIV human monoclonal antibodies reveals an immunodominant site in V2. PLoS One 8, e70859 (2013).

22. Hessell, A.J. et al. Multimeric epitope-scaffold HIV vaccines target V1V2 and differentially tune polyfunctional antibody responses. Cell Rep 28, 877-895 (2019).

23. Aiyegbo, M.S. et al. Peptide targeted by human antibodies associated with HIV vaccine-associated protection assumes a dynamic alpha-helical structure. PLoS One 12, e0170530 (2017).

24. Pancera, M. et al. Structure and immune recognition of trimeric pre-fusion HIV-1 Env. Nature 514, 455-461 (2014). 
25. Walker, L.M. et al. Broad and potent neutralizing antibodies from an African donor reveal a new HIV1 vaccine target. Science 326, 285-289 (2009).

26. Kimura, T., Wang, X.H., Williams, C., Zolla-Pazner, S. \& Gorny, M.K. Human monoclonal antibody 2909 binds to pseudovirions expressing trimers but not monomeric HIV-1 envelope proteins. Hum Antibodies 18, 35-40 (2009).

27. Gorny, M.K. et al. Identification of a new quaternary neutralizing epitope on human immunodeficiency virus type 1 virus particles. J Virol 79, 5232-5237 (2005).

28. Lee, J.H. et al. A broadly neutralizing antibody targets the dynamic HIV envelope trimer apex via a long, rigidified, and anionic beta-hairpin structure. Immunity 46, 690-702 (2017).

29. Sok, D. et al. Recombinant HIV envelope trimer selects for quaternary-dependent antibodies targeting the trimer apex. Proc Natl Acad Sci U S A 111, 17624-17629 (2014).

30. Cale, E.M. et al. Virus-like particles identify an HIV V1V2 apex-binding neutralizing antibody that lacks a protruding loop. Immunity 46, 777-791 (2017).

31. Doria-Rose, N.A. et al. New member of the V1V2-directed CAP256-VRC26 lineage that shows increased breadth and exceptional potency. J Viro/ 90, 76-91 (2016).

32. Doria-Rose, N.A. et al. Developmental pathway for potent V1V2-directed HIV-neutralizing antibodies. Nature 509, 55-62 (2014).

33. Wang, H. et al. Asymmetric recognition of HIV-1 Envelope trimer by V1V2 loop-targeting antibodies. elife 6, e27389 (2017).

34. Upadhyay, C. et al. Distinct mechanisms regulate exposure of neutralizing epitopes in the V2 and V3 loops of HIV-1 envelope. J Viro/ 88, 12853-12865 (2014).

35. Mayr, L.M. et al. Non-neutralizing antibodies targeting the V1V2 domain of HIV exhibit strong antibody-dependent cell-mediated cytotoxic activity. Sci Rep 7, 12655 (2017).

36. Musich, T. et al. Monoclonal antibodies specific for the V2, V3, CD4-binding site, and gp41 of HIV-1 mediate phagocytosis in a dose-dependent manner. J Viro/ 91, e02325-02316 (2017).

37. Zolla-Pazner, S. et al. Rationally Designed Vaccines Targeting the V2 Region of HIV-1 gp120 Induce a Focused, Cross-Clade-Reactive, Biologically Functional Antibody Response. J Viro/ 90, 10993-11006 (2016).

38. Balachandran, N., Bacchetti, S. \& Rawls, W.E. Protection against lethal challenge of BALB/c mice by passive transfer of monoclonal antibodies to five glycoproteins of herpes simplex virus type 2. Infect Immun 37, 1132-1137 (1982). 
39. Gorander, S., Ekblad, M., Bergstrom, T. \& Liljeqvist, J.A. Anti-glycoprotein g antibodies of herpes simplex virus 2 contribute to complete protection after vaccination in mice and induce antibodydependent cellular cytotoxicity and complement-mediated cytolysis. Viruses 6, 4358-4372 (2014).

40. Petro, C. et al. Herpes simplex type 2 virus deleted in glycoprotein D protects against vaginal, skin and neural disease. eLife 4, e02325-02316 (2015).

41. DiLillo, D.J., Tan, G.S., Palese, P. \& Ravetch, J.V. Broadly neutralizing hemagglutinin stalk-specific antibodies require FcgammaR interactions for protection against influenza virus in vivo. Nat Med 20, 143151 (2014).

42. Patterson, L.J. et al. Protection against mucosal simian immunodeficiency virus SIV(mac251) challenge by using replicating adenovirus-SIV multigene vaccine priming and subunit boosting. $J$ Virol 78, 2212-2221 (2004).

43. Gomez-Roman, V.R. et al. Vaccine-elicited antibodies mediate antibody-dependent cellular cytotoxicity correlated with significantly reduced acute viremia in rhesus macaques challenged with SIVmac251. J Immunol 174, 2185-2189 (2005).

44. Forthal, D.N. \& Moog, C. Fc receptor-mediated antiviral antibodies. Curr Opin Hiv Aids 4, 388-393 (2009).

45. Alpert, M.D. et al. ADCC develops over time during persistent infection with live-attenuated SIV and is associated with complete protection against SIV(mac)251 challenge. PLoS Pathog 8, e1002890 (2012).

46. Fouts, T.R. et al. Balance of cellular and humoral immunity determines the level of protection by HIV vaccines in rhesus macaque models of HIV infection. Proc Natl Acad Sci U S A 112, e992-999 (2015).

47. Barouch, D.H. et al. Protective efficacy of a global HIV-1 mosaic vaccine against heterologous SHIV challenges in rhesus monkeys. Cell 155, 531-539 (2013).

48. Vargas-Inchaustegui, D.A. \& Robert-Guroff, M. Fc receptor-mediated immune responses: new tools but increased complexity in HIV prevention. Curr HIV Res 11, 407-420 (2013).

49. Santra, S. et al. Human non-neutralizing HIV-1 envelope monoclonal antibodies limit the number of founder viruses during SHIV mucosal infection in rhesus macaques. PLoS Pathog 11, e1005042 (2015).

50. Henry Dunand, C.J. et al. Both neutralizing and non-neutralizing human H7N9 Influenza vaccineinduced monoclonal antibodies confer protection. Cell Host Microbe 19, 800-813 (2016).

51. Anand, S.P. et al. Longitudinal analysis of humoral immunity against SARS-CoV-2 Spike in convalescent individuals up to 8 months post-symptom onset. Cell Rep Med 2, 100290 (2021). 
52. Tauzin, A. et al. A single dose of the SARS-CoV-2 vaccine BNT162b2 elicits Fc-mediated antibody effector functions and T cell responses. Cell Host Microbe 29, 1137-1150 (2021).

53. Malherbe, D.C. et al. Combination adenovirus and protein vaccines prevent infection or reduce viral burden after heterologous clade $\mathrm{C}$ simian-human immunodeficiency virus mucosal challenge. $J$ Virol 92, e01092-01017 (2018).

54. Jones, A.T. et al. A trimeric HIV-1 envelope gp120 immunogen induces potent and broad anti-V1V2 loop antibodies against HIV-1 in rabbits and rhesus macaques. $J$ Viro/ 92, e01796-01717 (2018).

55. Jiang, X. et al. Rationally designed immunogens targeting HIV-1 gp120 V1V2 induce distinct conformation-specific antibody responses in rabbits. J Virol 90, 11007-11019 (2016).

56. Devasundaram, S. et al. Priming with DNA expressing trimeric HIV V1V2 alters the immune hierarchy favoring the development of V2-specific antibodies in rhesus macaques. J Viro/ 95, e0119301120 (2020).

57. Gorny, M.K. et al. Human anti-V2 monoclonal antibody that neutralizes primary but not laboratory isolates of human immunodeficiency virus type $1 . J$ Viro/ 68, 8312-8320 (1994).

58. Pinter, A. et al. The V1/V2 domain of gp120 is a global regulator of the sensitivity of primary human immunodeficiency virus type 1 isolates to neutralization by antibodies commonly induced upon infection. $J$ Virol 78, 5205-5215 (2004).

59. Nyambi, P.N. et al. Conserved and exposed epitopes on intact, native, primary human immunodeficiency virus type 1 virions of group M. J Viro/ 74, 7096-7107 (2000).

60. Gorny, M.K., VanCott, T.C., Williams, C., Revesz, K. \& Zolla-Pazner, S. Effects of oligomerization on the epitopes of the human immunodeficiency virus type 1 envelope glycoproteins. Virology 267, 220-228 (2000).

61. Rao, M. et al. Liposome-encapsulated human immunodeficiency virus-1 gp120 induces potent V1V2-specific antibodies in humans. J Infect Dis 218, 1541-1550 (2018).

62. Hessell, A.J. et al. Virus control in vaccinated rhesus macaques is associated with neutralizing and capturing antibodies against the SHIV challenge virus but not with V1V2 vaccine-induced anti-V2 antibodies alone. J Immunol 206, 1266-1283 (2021).

63. Powell, R.L. et al. An HIV vaccine targeting the V2 region of the HIV envelope induces a highly durable polyfunctional Fc-mediated antibody response in rhesus macaques. $J$ Viro/ 94 , e01175-01120 (2020).

64. Palker, T.J. et al. A conserved region at the $\mathrm{COOH}$ terminus of human immunodeficiency virus gp120 envelope protein contains an immunodominant epitope. Proc Natl Acad Sci U S A 84, 2479-2483 
(1987).

65. Hessell, A.J. et al. Reduced cell-associated DNA and improved viral control in macaques following passive transfer of a single anti-V2 monoclonal antibody and repeated simian/human immunodeficiency virus challenges. J Viro/ 92, e02198-02117 (2018).

66. Ackerman, M.E., Barouch, D.H. \& Alter, G. Systems serology for evaluation of HIV vaccine trials. Immunol Rev 275, 262-270 (2017).

67. Li, J. et al. HIV/SIV DNA vaccine combined with protein in a co-immunization protocol elicits highest humoral responses to envelope in mice and macaques. Vaccine 31, 3747-3755 (2013).

68. Kwong, P.D. \& Mascola, J.R. HIV-1 vaccines based on antibody identification, B cell ontogeny, and epitope structure. Immunity 48, 855-871 (2018).

69. Silva de Castro, I. et al. Anti-V2 antibodies virus vulnerability revealed by envelope V1 deletion in HIV vaccine candidates. iScience 24, 102047 (2021).

70. Rolland, M. et al. Increased HIV-1 vaccine efficacy against viruses with genetic signatures in Env V2. Nature 490, 417-420 (2012).

71. Barouch, D.H. et al. Vaccine protection against acquisition of neutralization-resistant SIV challenges in rhesus monkeys. Nature 482, 89-93 (2012).

72. Pegu, P. et al. Antibodies with high avidity to the gp120 envelope protein in protection from simian immunodeficiency virus SIV(mac251) acquisition in an immunization regimen that mimics the RV-144 Thai trial. J Viro/ 87, 1708-1719 (2013).

73. Singh, S. et al. Control of heterologous SIVsmE660 infection by DNA and protein co-immunization regimens combined with different toll-like receptor-4 (TLR-4) based adjuvants in macaques. $J$ Virol 92, e00281-00218 (2018).

74. Roederer, M. et al. Immunological and virological mechanisms of vaccine-mediated protection against SIV and HIV. Nature 505, 502-508 (2014).

75. Vaccari, M. et al. Adjuvant-dependent innate and adaptive immune signatures of risk of SIVmac251 acquisition. Nat Med 22, 762-770 (2016).

76. Vaccari, M. et al. Corrigendum: Adjuvant-dependent innate and adaptive immune signatures of risk of SIVmac251 acquisition. Nat Med 22, 1192 (2016).

77. Gordon, S.N. et al. Boosting of ALVAC-SIV vaccine-primed macaques with the CD4-SIVgp120 fusion protein elicits antibodies to V2 associated with a decreased risk of SIVmac251 acquisition. $J$ Immunol 197, 2726-2737 (2016). 
78. Om, K. et al. Adjuvanted HIV-1 vaccine promotes antibody-dependent phagocytic responses and protects against heterologous SHIV challenge. PLoS Pathog 16, e1008764 (2020).

79. Landais, E. et al. Broadly neutralizing antibody responses in a large longitudinal sub-saharan HIV primary infection cohort. PLoS Pathog 12, e1005369 (2016).

80. Kayman, S.C. et al. Presentation of native epitopes in the V1/V2 and V3 regions of human immunodeficiency virus type $1 \mathrm{gp} 120$ by fusion glycoproteins containing isolated gp120 domains. J Virol 68, 400-410 (1994).

81. McKeating, J.A. et al. Immunogenicity of full length and truncated forms of the human immunodeficiency virus type I envelope glycoprotein. Immunol Lett 51, 101-105 (1996).

82. Israel, Z.R., Gorny, M.K., Palmer, C., McKeating, J.A. \& Zolla-Pazner, S. Prevalence of a V2 epitope in clade $B$ primary isolates and its recognition by sera from HIV-1-infected individuals. AIDS 11, 128-130 (1997).

83. Liu, L. et al. Anti-V2 antibody deficiency in individuals infected with HIV-1 in Cameroon. Virology 529, 57-64 (2019).

84. Bekker, L.G. et al. Subtype C ALVAC-HIV and bivalent subtype C gp120/MF59 HIV-1 vaccine in lowrisk, HIV-uninfected, South African adults: a phase 1/2 trial. Lancet HIV 5, e366-e378 (2018).

85. Gray, G.E. et al. Immune correlates of the Thai RV144 HIV vaccine regimen in South Africa. Sci Transl Med 11, eaax1880 (2019).

86. Moore, P.L., Gorman, J., Doria-Rose, N.A. \& Morris, L. Ontogeny-based immunogens for the induction of V2-directed HIV broadly neutralizing antibodies. Immunol Rev 275, 217-229 (2017).

87. Karasavvas, N. et al. The Thai Phase III HIV Type 1 Vaccine trial (RV144) regimen induces antibodies that target conserved regions within the V2 loop of gp120. AIDS Res Hum Retroviruses 28, 1444-1457 (2012).

88. Gorny, M.K. et al. Functional and immunochemical cross-reactivity of V2-specific monoclonal antibodies from HIV-1-infected individuals. Virology 427, 198-207 (2012).

89. Redd, A.D., Quinn, T.C. \& Tobian, A.A. Frequency and implications of HIV superinfection. Lancet Infect Dis 13, 622-628 (2013).

90. Chung, A.W. et al. Identification of antibody glycosylation structures that predict monoclonal antibody Fc-effector function. AIDS 28, 2523-2530 (2014).

91. Zolla-Pazner, S. et al. Serotyping of primary human immunodeficiency virus type 1 isolates from diverse geographic locations by flow cytometry. J Viro/ 69, 3807-3815 (1995). 
92. Gorny, M.K. et al. Preferential use of the VH5-51 gene segment by the human immune response to code for antibodies against the V3 domain of HIV-1. Mol Immunol 46, 917-926 (2009).

93. Malherbe, D.C. et al. Modified adenovirus prime-protein boost clade C HIV vaccine strategy results in reduced viral DNA in blood and tissues following Tier 2 SHIV challenge. Front Immunol 11, 626464 (2020).

94. R Studio Team. RStudio: Integrated Development for R., RStudio Inc., http://www.rstudio.com/. (2015).

95. R Development Core Team. 2013. R: A language and environment for statistical computing R Foundation for Statistical Computing, Vienna, Austria.

96. Siddappa, N.B. et al. R5 clade C SHIV strains with tier 1 or 2 neutralization sensitivity: tools to dissect env evolution and to develop AIDS vaccines in primate models. PLoS One 5, e11689 (2010).

\section{Table}

Table I. HIV-1 vaccine regimens studied in M. mulatta

\begin{tabular}{|c|c|c|c|c|c|}
\hline $\begin{array}{l}\text { Immunization } \\
\text { Designation }\end{array}$ & $\begin{array}{c}\text { Prime } \\
\text { (Env/gag genes) }\end{array}$ & Protein Boost & Adjuvant & Schedule & References \\
\hline DNA + gp120 & $\begin{array}{c}\text { DNA } \\
\text { (AE/92TH023 } \\
\text { gp160 and SIV gag) }\end{array}$ & $\begin{array}{c}\text { gp120 } \\
(B / M N+A E / A 244)\end{array}$ & Adjuplex & $\begin{array}{ll}\text { Wk 0: } & \text { DNA + gp120s } \\
\text { Wk 4: } & \text { DNA + gp120s } \\
\text { Wk 12: DNA + gp120s } \\
\text { Wk 20: DNA + gp120s }\end{array}$ & 62 \\
\hline SAd7 + gp140 & $\begin{array}{l}\text { Simian Adenovirus } 7 \\
\text { (SAd7 includes } \\
\text { transgene for C/1086 } \\
\text { gp150 HIV-1 Env and } \\
\text { SIVmac239 Gag) }\end{array}$ & $\begin{array}{l}\text { gp140 } 141088 \text { trimer + } \\
\text { GBV-C E2* }\end{array}$ & Adjuplex & $\begin{array}{l}\text { Wk 0: SAd7 } \\
\text { Wk 4: SAd7 + protein } \\
\text { Wk 16: Protein }\end{array}$ & 53 \\
\hline $\begin{array}{c}\text { DNA + } \\
\text { V1V2-scaffold }\end{array}$ & $\begin{array}{c}\text { DNA } \\
(\mathrm{C} / \mathrm{ZM} 53 \mathrm{gp} 120)\end{array}$ & $\begin{array}{l}\text { V1V2 } 2_{C Z Z M 53}-2 F 5 K \\
\text { V1V2 } 2_{C Z Z 109} \text {-TTB } \\
\text { V1V2 } 2_{A E / A 244}-2 J 9 C\end{array}$ & Adjuplex & $\begin{array}{cc}\text { Wk 0: } & \begin{array}{c}\text { DNA + V1V2-scaffold } \\
\text { cocktail } \\
\text { DNA + V1V2-scaffold } \\
\text { cocktail }\end{array} \\
\text { Wk 8: } \\
\text { Wk 20: } \begin{array}{c}\text { DNA + V1V2-scaffold } \\
\text { cocktail }\end{array}\end{array}$ & 22 \\
\hline
\end{tabular}

*human pegivirus GBV-C E2 glycoprotein

\section{Figures}




\begin{tabular}{|c|c|c|c|c|c|c|c|c|c|c|c|c|c|}
\hline & $\mathrm{mAb}$ & CH58 & CH59 & $\begin{array}{c}\text { CAP228 } \\
19 \mathrm{~F}\end{array}$ & $\begin{array}{c}\text { CAP228 } \\
\text { 3D. } 1\end{array}$ & $\begin{array}{c}\text { CAP228 } \\
16 \mathrm{H}\end{array}$ & $830 \mathrm{~A}$ & 697-30D & 2158 & 1361 & PG9 & PGT145 & $447-52 D$ \\
\hline & Specificity & & & V2p & & & & $v$ & & & $v_{2} q$ & V2qt & V3 \\
\hline \multicolumn{14}{|l|}{ Antigen } \\
\hline \multirow{7}{*}{ gp120 } & $\mathrm{gp} 120_{\text {C/ZM53 }}$ & 3.18 & 0.00 & 7.22 & 6.46 & 6.94 & 6.66 & 5.06 & 5.86 & 6.19 & 2.08 & 0.00 & 6.67 \\
\hline & $\mathrm{gp}_{120_{0 / n u z}}$ & 0.01 & 0.01 & 0.02 & 0.00 & 0.02 & 5.49 & 3.55 & 4.06 & 3.93 & 0.04 & 0.00 & 9.82 \\
\hline & gp120 Alausses & 0.45 & 0.01 & 3.95 & 3.11 & 3.64 & 3.52 & 0.67 & 1.47 & 1.58 & 0.11 & 0.00 & 7.62 \\
\hline & $\mathbf{V 3}_{\text {cyeonsc }}$ & 0.12 & 0.03 & 0.02 & 0.02 & 0.07 & 0.07 & 0.15 & 0.04 & 0.04 & 0.00 & 0.00 & 8.90 \\
\hline & CS cusve _. . - & 0.13 & 0.04 & 0.07 & 0.04 & 0.15 & 0.03 & 0.02 & 0.02 & 0.03 & 0.00 & 0.00 . & 0.01 \\
\hline & 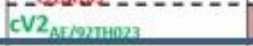 & 5.70 & 6.51 & 6.20 & 4.63 & 6.68 & 0.37 & 0.04 & 0.01 & 0.01 & 0.00 & 0.00 & 0.01 \\
\hline & cV2 ${ }_{\text {CruM109 }}$ & 0.09 & 8.32 & 0.03 & 0.01 & 0.06 & 2.48 & 0.06 & 0.00 & 2.79 & 0.00 & 0.00 & 0.03 \\
\hline \multirow{6}{*}{ Pentides } & $\mathrm{CV} / 2_{\mathrm{C} / 1086}$ & 6.69 & 7.79 & 7.89 & 6.50 & 7.66 & 1.23 & 0.63 & $0.10^{\circ}$ & 0.03 & $0.00^{\circ}$ & 0.00 & 0.03 \\
\hline & $\mathrm{CV} z_{\mathrm{B} / \mathrm{Nuz}}$ & 0.26 & 0.35 & 0.14 & 0.05 & 0.18 & 0.01 & 0.07 & 0.00 & 0.00 & 0.00 & $0.00^{\circ}$ & 0.03 \\
\hline & $\mathrm{cV}_{\text {Almoses }}$ & 1.73 & 0.24 & 4.81 & 3.14 & 6.99 & 0.00 & 0.13 & 0.00 & 0.00 & 0.00 & 0.00 & 0.02 \\
\hline & V2(AA166-185) & 9.21 & 9.73 & 10.26 & 9.64 & 10.73 & 0.01 & 0.10 & 0.00 & 0.00 & 0.00 & 0.00 & 0.04 \\
\hline & V2(AA172-191) & 9.11 & 9.29 & 10.31 & 8.00 & 11.23 & 0.01 & 0.04 & 0.00 & 0.00 & 0.00 & 0.00 & 0.03 \\
\hline & V2(AA190-209) & 0.57 & 0.90 & 0.19 & 0.05 & 0.22 & 0.01 & 0.05 & 0.00 & 0.00 & 0.00 & 0.00 & 0.03 \\
\hline \multirow{3}{*}{ V1V2-tags } & V1V2 $2_{\text {AV/M244 }}$-tags & 4.13 & 5.45 & 3.97 & 4.21 & 4.00 & 3.32 & 0.40 & 2.32 & 1.72 & 0.03 & 0.00 & 0.00 \\
\hline & $V_{1 V 2}$ C1086-tags & 6.05 & 7.29 & 7.18 & 5.88 & 7.02 & 0.83 & 0.01 & 0.06 & 0.79 & 0.00 & 0.00 & 0.00 \\
\hline & VIV2 & 0.01 & 0.00 & 0.23 & 0.01 & 0.21 & 4.72 & 3.92 & 4.37 & 4.59 & 0.00 & 0.00 & 0.00 \\
\hline \multirow{4}{*}{ V1V2-gp70s } & V1V2 [/97ZAO12 - Bp70 & 0.01 & 0.69 & 7.63 & 1.52 & 7.07 & 5.87 & 5.82 & 6.31 & 6.57 & 0.00 & 0.00 & 0.00 \\
\hline & 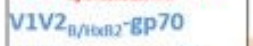 & 0.01 & 0.00 & 0.78 & 0.01 & 0.57 & 6.34 & 5.61 & 6.08 & 7.45 & 0.00 & 0.00 & 0.00 \\
\hline & 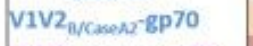 & 1.04 & 0.00 & 3.51 & 1.80 & 3.74 & 5.27 & 4.35 & 5.43 & 5.58 & 0.00 & 0.00 & 0.00 \\
\hline & V1V2, & 8.87 & 3.30 & 9.01 & 9.23 & 9.23 & 7.78 & 0.05 & 4.68 & 6.53 & 0.00 & 0.00 & 0.00 \\
\hline & VIV2 $2_{\text {AF/A2A4 }}-1$ FD6 & 3.18 & 3.35 & 5.20 & 4.30 & 6.76 & 3.82 & 2.64 & 2.82 & 2.63 & 0.32 & 0.00 & 0.01 \\
\hline \multirow{3}{*}{ V1V2-1FD6s } & V1V2 cramios $^{-1 F D 6}$ & 0.01 & 0.01 & 0.00 & 0.01 & 0.01 & 7.06 & 0.03 & 6.40 & 7.60 & 0.37 & 0.00 & 0.00 \\
\hline & $V 1 V z_{n / m u r^{-1 F D 6}}$ & 0.04 & 0.04 & 0.03 & 0.03 & 0.03 & 4.90 & 3.70 & 4.45 & 4.69 & 0.00 & 0.03 & 0.03 \\
\hline & $\mathrm{V}_{1 \mathrm{~V} 2_{\mathrm{AMG}}}-1 \mathrm{FD} 6$ & 0.03 & 0.00 & 0.18 & 0.38 & 0.19 & 0.63 & 0.00 & 0.00 & 0.01 & 0.00 & 0.00 & 0.00 \\
\hline
\end{tabular}

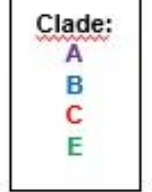

Binding in AUC

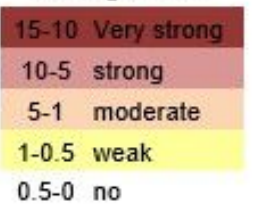

b

V $2 p$ mAbs

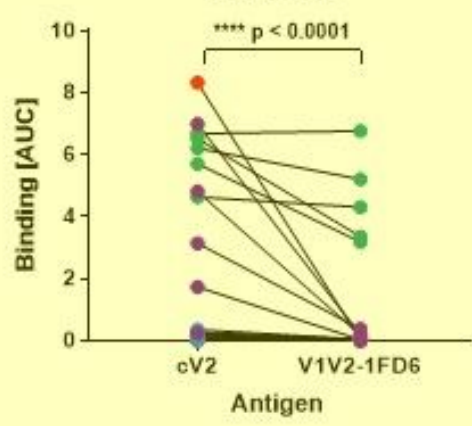

V2p mAbs vs. 92TH023/A244

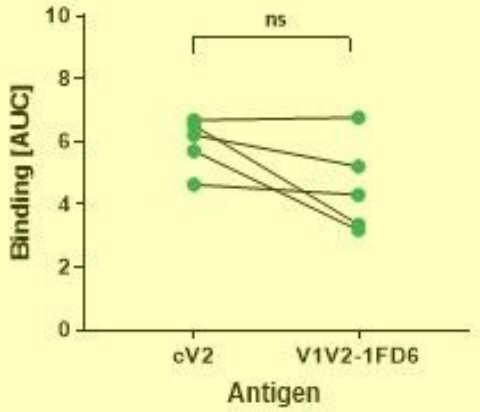

V2i mAbs

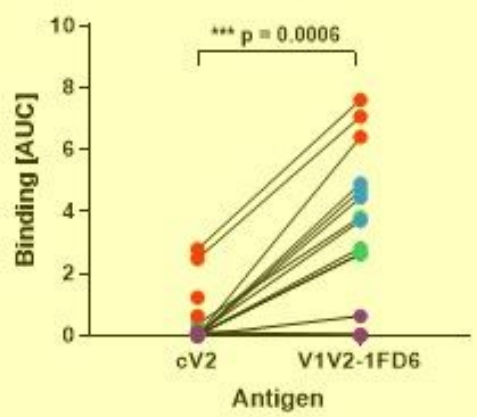

Figure 1

Reactivity of HIV-1 Env antigens with V2 monoclonal antibodies. a A multiplex bead binding assay was used to determine the levels of reactivity of mAbs specific for different V2 epitopes with various HIV-1 antigens. The clade from which each antigen was derived is indicated by the subscripted letter and by color coding. As a control, V3 mAb 447-52D was used. Irrelevant mAbs and PBS were used as negative controls in each experiment (not shown). Data are shown as AUC values generated from titration curves for each mAb, provided in Supplementary Figure S1. Strength of binding is color-coded as per the spectrum shown in the figure. Experiments were performed at least twice. b Statistical analyses comparing binding to cV2 peptides and V1V2-1FD6 proteins by V2p mAbs (left graph) and V2i mAbs (right graph) are shown along with a comparison of V2p mAbs vs cV2 peptides and V1V2-1FD6 proteins 
derived from HIVAE/92TH023 and HIVAE/A244 (center graph). Color of symbols show the clade of the antigen used as per those used in Figure 1a. Lines connect pairs of a given mAb with reagents from a given strain. For example, the connected red symbols in the left panel of Figure $1 \mathrm{~b}$ represent the reactivity of V2p mAb CH59 with cV2C/ZM109 and V1V2C/ZM109-1FD6. Statistical analyses were performed with the two-tailed Wilcoxon matched pairs signed rank test and are shown for: all HIV-1 strains used in Figure1b (left and right graphs) or for V2p mAbs reacting to the HIV-1 strain A244 (middle graph). AA: amino acid; Ab: antibody; Ags: antigens; AUC: area-under-the-curve; consC: consensus C; cV2: cyclic V2; Env: envelope; mAb: monoclonal antibody. 
a

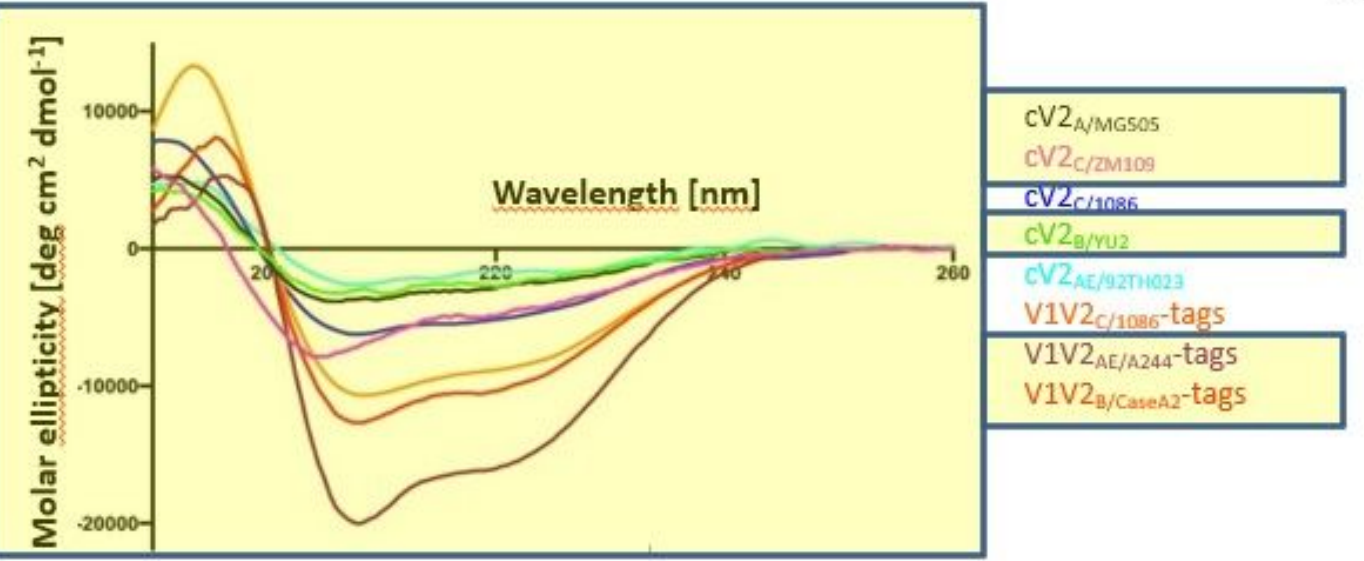

b

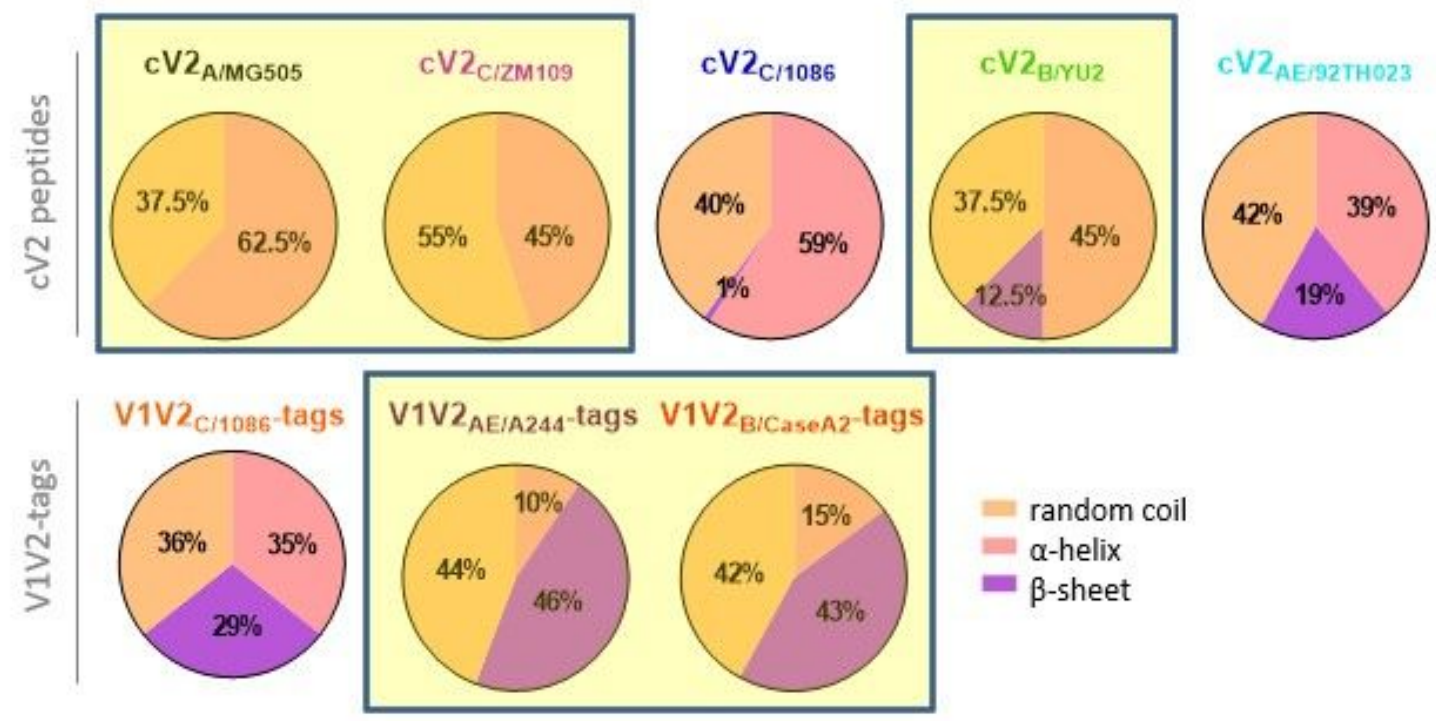

C

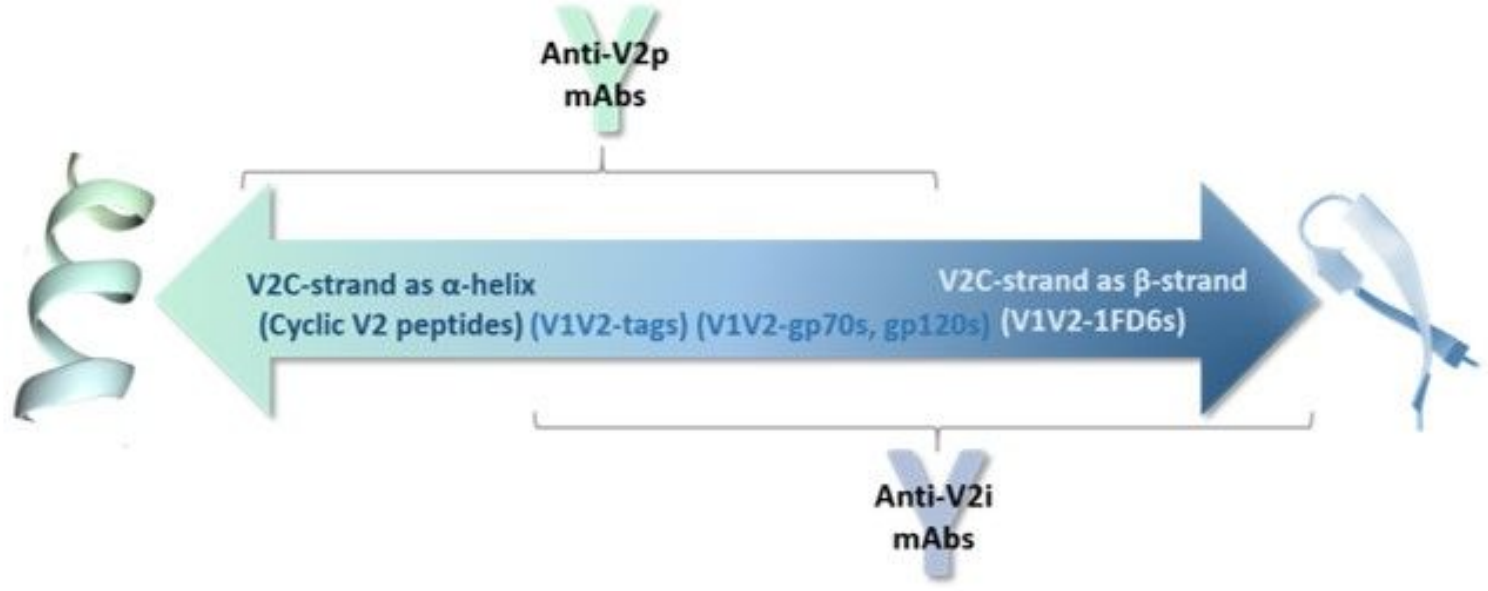

\section{Figure 2}

Structural conformations of the V1V2 domain. a Circular dichroism spectra of five cyclic V2 peptides and three V1V2-tags proteins. b Pie charts depicting the percentage of secondary structure (random coil [gold], a-helix [salmon], or $\beta$-sheet [purple]) determined by the circular dichroism spectra and calculated by CDFIT software (https://www.ruppweb.org/Xray/comp/cdfit.htm). c Schematic diagram showing the alterative structures of the V2 C-strand (V2C) between an a-helix (left) and a $\beta$-strand (right). According to 
the proportion of each molecule present in the two conformations, the antigens will react preferentially with V2p Abs (left), V2i Abs (right), or both (middle). Abbreviations as in Figure 1.

Figure 3

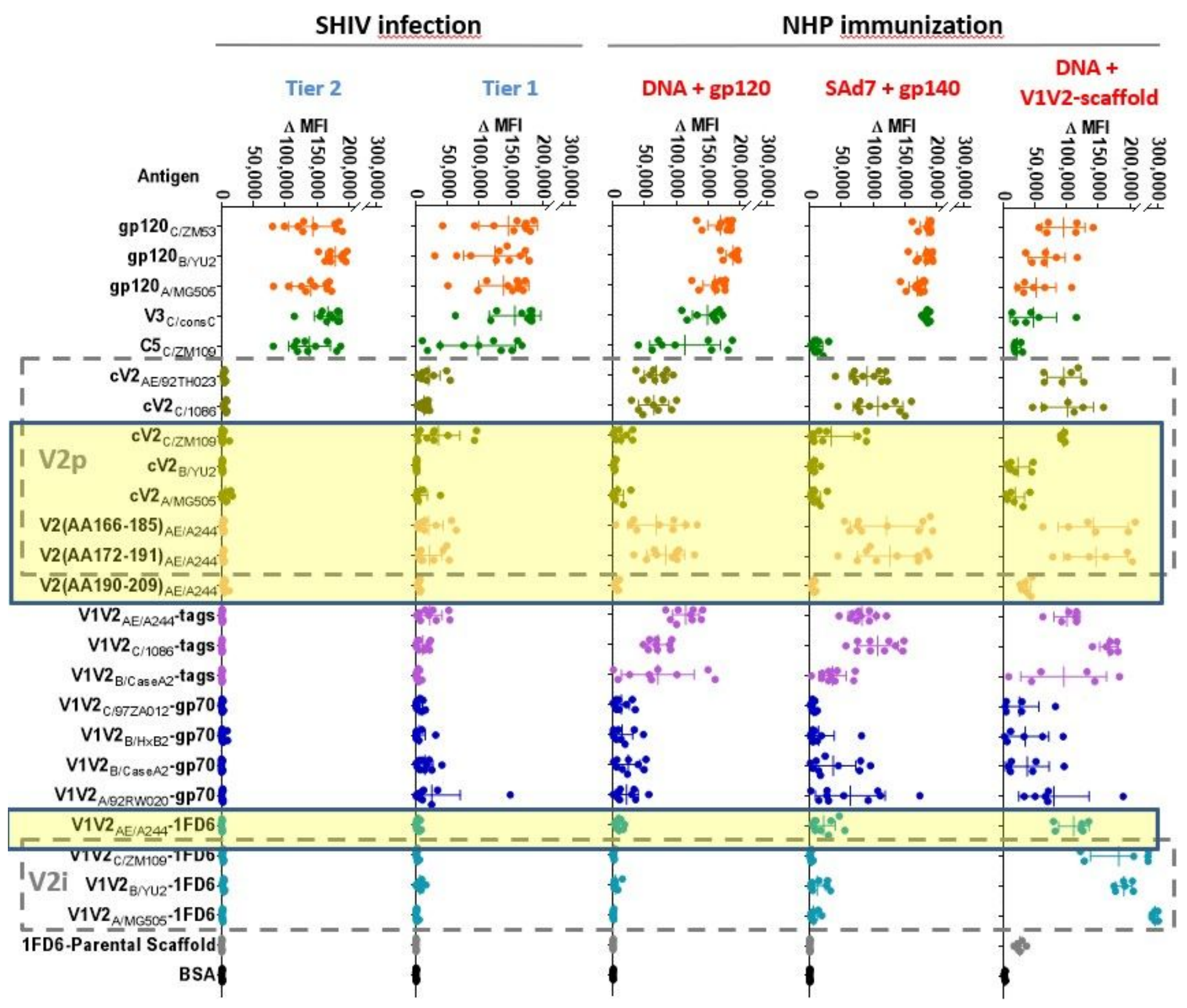

Figure 3

Dot plot of $A b$ binding activities in plasma from infected and immunized NHPs assessed in the multiplex bead Ab binding assay. Columns from left to right: Tier 2 SHIVC/1157-ipd3N4-infected NHPs; Tier 1 SHIVC/1157ipEL-p-infected NHPs; NHPs immunized with "DNA + gp120"; NHPs immunized with "SAd7 + gp140"; NHPs immunized with "DNA + V1V2-scaffolds". Binding activity of plasma was measured against the same set of 24 Env antigens used in Figure 1a; each row lists a tested antigen. Boxes with 
dashed lines denote antigens reactive with V2p- or V2i-specific mAbs. Beads identified as "1FD6 parental scaffold" (devoid of the V1V2 insert) and BSA (bovine serum albumin) were used as negative controls. Each dot represents the normalized mean fluorescence intensity $(\triangle \mathrm{MFI})$ generated with a plasma specimen diluted 1:200 from a single animal. Colors of the dots represents a type of antigen (orange $=$ gp120s, dark green $=$ non-V1V2 linear peptides $(\mathrm{V} 3$ and C5), olive green $=\mathrm{cV} 2$ peptides, cream $=$ linear V2 peptides, purple $=$ V1V2-tags, dark blue $=$ V1V2-gp70s, light blue $=$ V1V2-1FD6s, gray $/$ black $=$ negative controls). Plasma samples from infected NHPs were drawn 18 and 11 weeks post last challenge for Tier 1 SHIV- and Tier 2 SHIV-infected NHPs, respectively. Specimens from immunized NHPs were drawn two weeks after the last immunization. A mAb pool was used as a positive Ab control (not shown). Intensity of the reactivity is shown as the mean MFI calculated from experiments normalized on the basis of the reactivity of the mAb pool in each experiment from which background (PBS-TB) was subtracted. Experiments were performed at least twice and in each experiment, samples were tested in duplicate. Abbreviations as in Figure 1. 


\section{V2p Abs}

V2i Abs
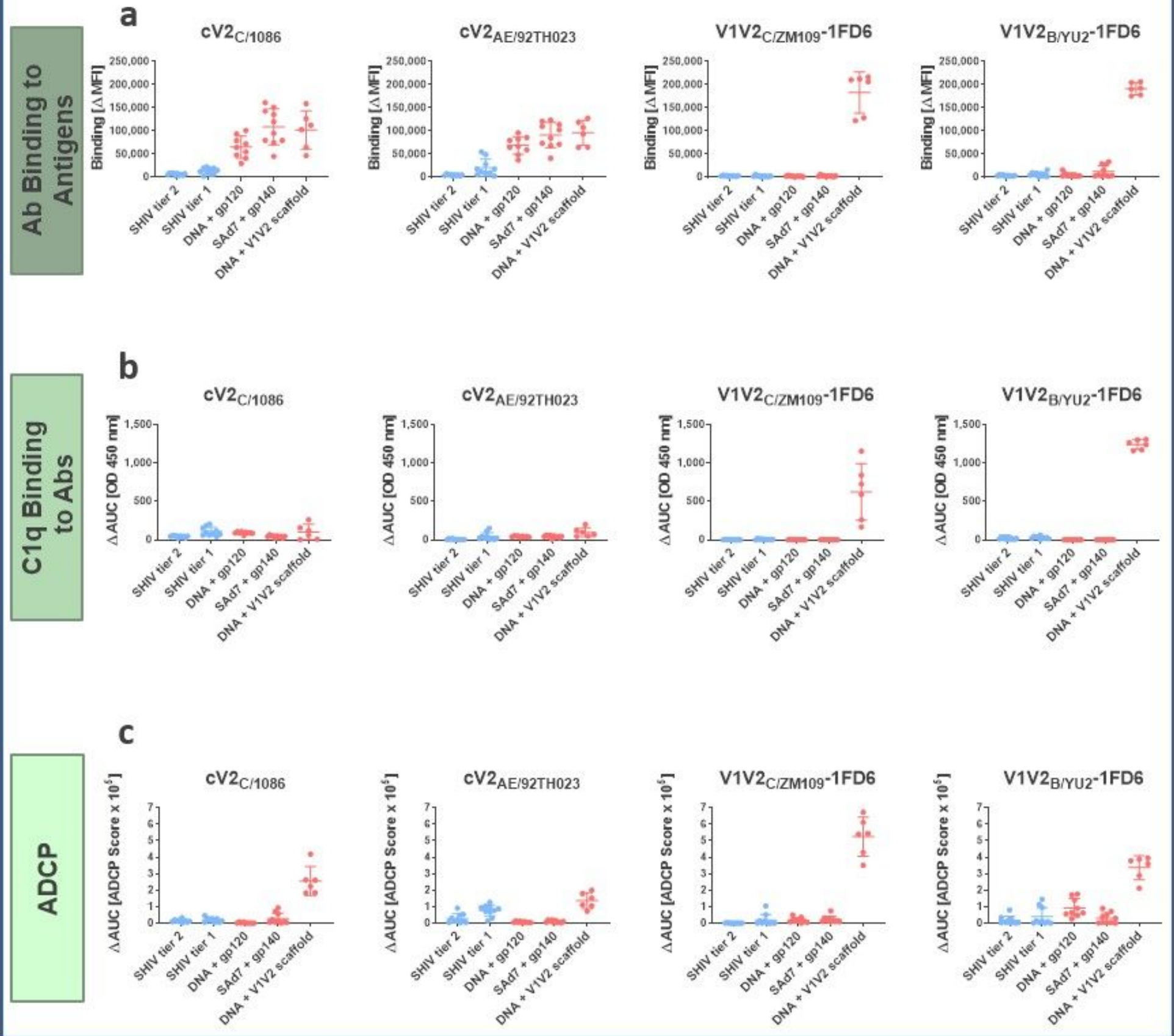

\section{Figure 4}

Antibody binding, C1q binding and ADCP responses of NHPs infected with SHIV (blue) or immunized with one of the three immunization regimens (red). Each panel shows results from a different assay: a multiplex bead $\mathrm{Ab}$ binding, $\mathrm{b}$ C1q binding, and $\mathrm{c} A D C P$. Activities were measured using $\mathrm{cV} 2$ peptides (reactive with V2p Abs) or V1V2-1FD6 scaffold proteins (reactive with V2i Abs). Binding values in panel a are $\triangle \mathrm{MFI}$ as shown in Figure 3. Values in panels $\mathrm{b}$ and $\mathrm{c}$ represent area-under-the-curve $(\triangle \mathrm{AUC})$ and were calculated for $\mathrm{C} 1 \mathrm{q}$ from two-fold plasma titrations ranging from 1:50-400, and for ADCP from five-fold dilutions ranging from 1:50 - 781,250 (Supplementary Figure S4). $\triangle A U C$ values were calculated as the 
AUC from each NHP group two weeks after immunization or 11 (SHIV Tier 2) or 18 (SHIV Tier 1) weeks after infection from which the pre-bleed background response was subtracted. The mean of all animals within each group is indicated. C1q titrations were performed once and ADCP experiments were performed at least twice.

Figure 5

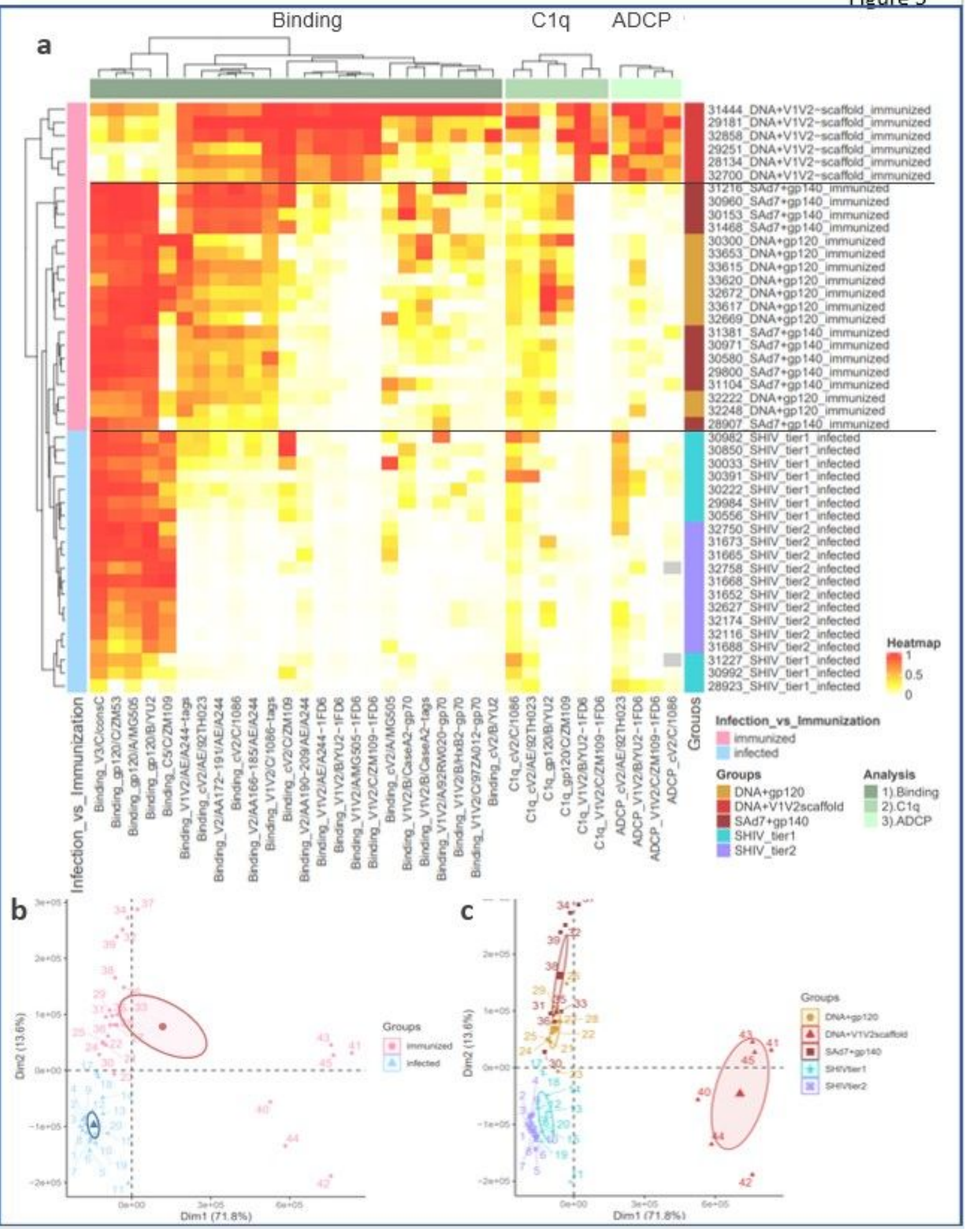

Figure 5 
Analysis of data from all 45 animals and all three types of assays. a Heatmap summarizing the humoral immune responses in immunized versus SHIV-infected NHPs. Immune responses shown in Figures 3 and 4 and Supplementary Figures S2-S4 were normalized and color-coded as indicated in the heatmap legend. Columns represent immune responses grouped according to $\mathrm{Ab}$ binding, $\mathrm{C} 1 \mathrm{q}$ binding and $\mathrm{ADCP}$ and are clustered within each parameter ( $A b$ binding, $C 1 q$ binding or $A D C P$ ) measured using the antigens shown at the bottom of the heatmap. Rows represent single animals, each identified by number, vaccine regimen or SHIV-strain used for infection. The heatmap is shown with hierarchical clustering according to the three dendrograms at the top and the one to the left. b, c Principal component analyses were performed using all values from panel a and are colored in panel $b$ as immunized (pink) versus infected (blue), or in panel c, according to NHP groups as indicated in the legend. The ellipses indicate $95 \%$ confidence interval of the means. 


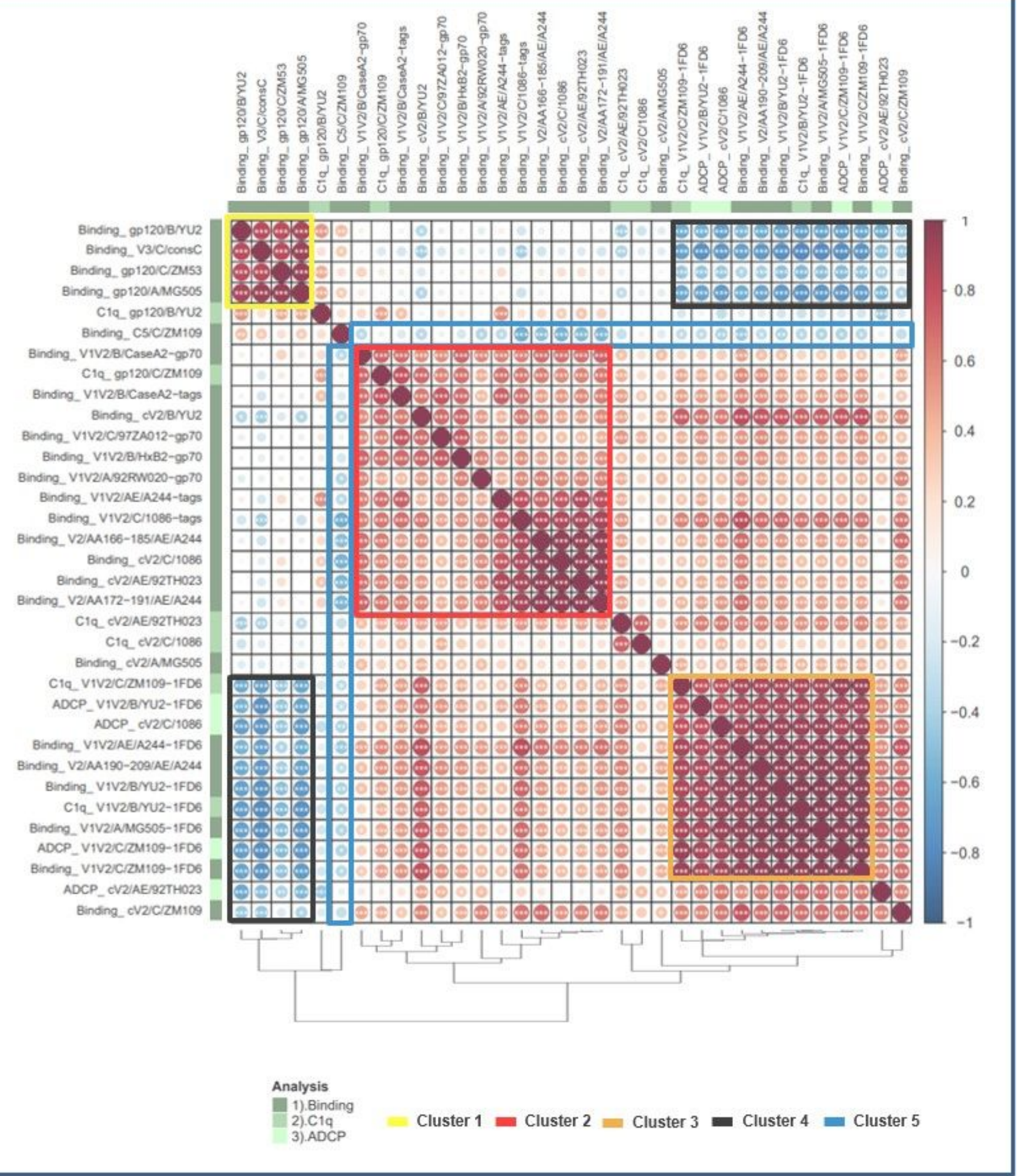

\section{Figure 6}

Correlation analysis of Ab binding, C1q binding and ADCP against various HIV-1 Env antigens. Correlogram summarizing pairwise correlations among indicated parameters for all 45 studied NHPs. In the correlogram, the magnitude of the correlation coefficient $r$ is color-coded as per the spectrum shown on the right with red colors representing positive and blue colors negative correlations between any two parameters. Asterisks within each cell indicate statistically significant correlations $\left({ }^{*} p<0.05\right.$, ${ }^{* *} p<$ 
$0.001, * \star \star p<0.005)$. The correlogram is shown with hierarchical clustering according to the dendrogram at the bottom. Correlation analysis was done using Pearson correlation. The five most prominent correlation clusters are highlighted with boxes and numbered as indicated in the legend.

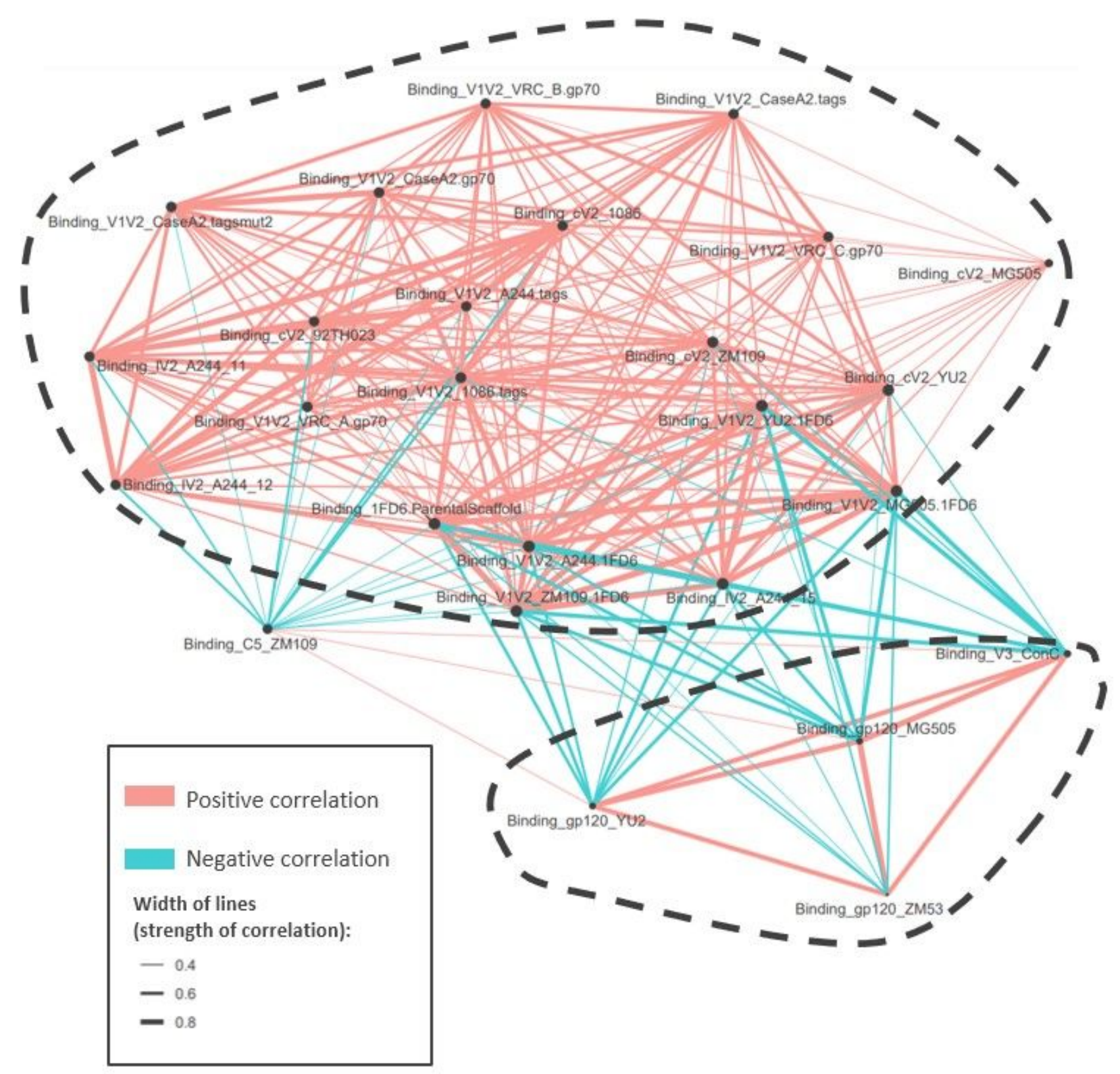

Figure 7

Correlation network analysis of Ab binding responses among all studied NHPs. Nodes represent Ab responses against each specific designated antigen, and red and blue lines represent positive and negative correlations between connected parameters, respectively. Only significant correlations $(p<0.05)$ are displayed. Line thickness corresponds to the strength of the correlation coefficient ( $r 2)$. Node size, 
indicated by the size of the black closed circles at each node, corresponds to the sum of correlations of the respective $A b$ response. The two most prominent correlation clusters are encircled with dashed lines.

\section{Supplementary Files}

This is a list of supplementary files associated with this preprint. Click to download.

- reportingsummary08132021.pdf

- NatCommSupplementary08132021.pdf

- editorialpolicychecklist08132021.pdf 